I. Kubo and H. Murata

Nagoya Math. J.

Vol. 81 (1981), 1-25

\title{
PERTURBED BILLIARD SYSTEMS II BERNOULLI PROPERTIES
}

\author{
IZUMI KUBO AND HIROSHI MURATA*)
}

\section{§1. Introduction}

One of the authors has shown the ergodicity of the perturbed billiard system which can describe the motion of a particle in a potential field of a special type [5], [6]. Since then, some development has been made, and we are now able to show the Bernoulli property of the system in this article. We hope, the result gives a new progress in statistical mechanics. Our method of the proof is inspired by the idea of D. S. Ornstein and B. Weiss [9], which has been used by G. Gallavotti and D. S. Ornstein [3] for a Sinai billiard system.

A perturbed billiard transformation will be prescribed in $\S 3$. Roughly speaking, it is an automorphism $T_{*}$ of two dimensional measure space $(M, \nu)$ which can be expressed as the product of $T_{1}$ and $T$, where $T_{1}$ is a $\nu$-preserving $C^{2}$-diffeomorphism of $M$ and where $T$ is a Sinai billiard transformation. Such an automorphism $T_{*}$ appears in a dynamical system of a particle moving in a potential field which is a composition of several finite range potentials (see [5], [6]). In order to discuss such a perturbed billiard system we need three assumptions $(\mathrm{H}-1) \sim(\mathrm{H}-3)$, which specify the diffeomorphism $T_{1}$. Under these assumptions, the perturbation of $T$ by $T_{1}$ is not so much. Details of them will be found in $\S 3$.

Our main results are the following:

THEOREM 2. Under the assumptions $(\mathrm{H}-1) \sim(\mathrm{H}-3)$, partitions $\alpha^{(c)}$ and $\alpha^{(e)}$ are weak Bernoulli generators for $T_{*}$. Thus $T_{*}$ is isomorphic to a Bernoulli shift.

Here $\alpha^{(c)}$ and $\alpha^{(e)}$ are partitions of $M$ whose elements are connected

Received June 7, 1975.

Revised December 4, 1979.

*) This author was partially supported by Yukawa Fellowship. 
components such that the restrictions of $T_{*}^{-1}$ and $T_{*}$ on them are continuous, respectively.

TheOREM 3. Under the assumptions $(\mathrm{H}-1) \sim(\mathrm{H}-3)$, every countable partition $\alpha$ is weakly Bernoullian for $T_{*}$, whenever $\log d(x ; \partial \alpha)$ is integrable.

Here $d(x ; \partial \alpha)$ is the distance between a point $x$ and the union $\partial \alpha$ of the boundaries of all elements of $\alpha$.

Theorem 5. Under the assumptions $(\mathrm{H}-1) \sim(\mathrm{H}-3)$ and $(\mathrm{f}-1) \sim(\mathrm{f}-3)$, if $\left\{S_{t}^{f}\right\}$ is a K-system and $\alpha$ is a finite partition such that $\log \tilde{d}(w ; \partial \alpha)$ is integrable, then $\alpha$ is very weakly Bernoullian for $\left\{S_{t}^{f}\right\}(t \neq 0)$. Furthermore $\left\{S_{t}^{f}\right\}$ is a Bernoulli flow.

As stated in Corollary 5.3, $\left\{S_{t}^{f}\right\}$ is a $K$-system if it does not have any point spectrum. With this result, we have a stronger assertion Corollary 6.2. Here $\left\{S_{t}^{f}\right\}$ is a flow of Kakutani-Ambrose type whose basic transformation and ceiling function are $T_{*}$ and $f(x)$, respectively. There we assume the conditions $(f-1) \sim(f-3)$, which are prescribed in $\S 5$, so as $f(x)$ to be regular. Actually, if $f(x)$ is positive and smooth on $M$, then they are obviously satisfied. Our formulation is complicated, but necessary in order to apply to the case of dynamical system on a potential field as described above.

In section 2 some lemmas to make easier checking weak Bernoulli property will be given. In section 3 some fundamental results of the perturbed billiard transformation $T_{*}$, which have been shown in [5], will be summarized. In section 4 the proofs of Theorem 2 and Theorem 3 will be shown appealing lemmas in $\S 2$. The most complicated parts of the proofs are in the estimations of densities of measures related to transversal fibres of $T_{*}$. In section 5 we will discuss on the construction of transversal fibres and the $K$-properties of the flow $\left\{S_{t}^{f}\right\}$. In section 6 the proof of Theorem 5 will be shown by using properties of the transversal fibres.

Lastly we remark that the same results can be obtained for more general $T_{1}$ as discussed in [6], since the properties stated in $\S 3$ are also true for the general case.

\section{§2. Weak and very weak Bernoulli partitions}

Let $(M, \nu)$ be a Lebesgue space with total mass $\nu(M)=1$, and let $T$ 
be a bimeasurable measure preserving transformation on $M$. D. S. Ornstein gave the following definitions:

Definition 2.1. A countable partition $\alpha$ of $M$ is said to be weakly Bernoullian for $T$, if for any $\varepsilon>0$ there exists $N \geq 0$ such that for all $N^{\prime \prime} \geq N^{\prime} \geq N$, all $n \geq 0$, and $\varepsilon$-a.e. $B$ in $\bigvee_{i=N^{\prime}}^{N^{\prime \prime}} T^{-i} \alpha$

$$
\sum_{\substack{n \in \vee^{i} \\ i=0}}|\nu(A)-\nu(A \mid B)|<\varepsilon .
$$

Here " $\varepsilon$-a.e. $B$ in $\xi$ " means "except element $B$ of the partition $\xi$ which is included in a set of measure $\varepsilon$ ". For two countable partitions $\alpha=\left\{A_{i}\right\}$ and $\beta=\left\{B_{i}\right\}$ of $M$, define the usual metric by

$$
d(\alpha, \beta) \equiv \sum_{i} \nu\left(A_{i} \ominus B_{i}\right),
$$

where $A \ominus B$ denotes the symmetric difference of the sets $A$ and $B$. For given two sequences of partitions $\left\{\alpha_{j}\right\}_{1}^{n}$ and $\left\{\beta_{j}\right\}_{1}^{n}$ write

$$
\left\{\alpha_{j}\right\}_{1}^{n} \sim\left\{\beta_{j}\right\}_{1}^{n}
$$

if for all $k_{j}, 1 \leq j \leq n$,

$$
\mu_{X}\left(\bigcap_{1}^{n} A_{k_{j}}^{(j)}\right)=\mu_{Y}\left(\bigcap_{1}^{n} B_{k_{j}}^{(j)}\right),
$$

where $\alpha_{j}=\left\{A_{1}^{(j)}, \cdots, A_{a(j)}^{(j)}\right\}$ are partitions on $\left(X, \mu_{X}\right)$ and $\beta_{j}=\left\{B_{1}^{(j)}, \cdots, B_{b(j)}^{(j)}\right\}$ are partitions on $\left(Y, \mu_{Y}\right)$. Further define the metric $\bar{d}$ by

$$
\bar{d}\left(\left\{\alpha_{j}\right\}_{1}^{n},\left\{\beta_{j}\right\}_{1}^{n}\right)=\inf _{\left\{\bar{\alpha}_{j}\right\},\left\{\bar{\beta}_{j}\right\}} \frac{1}{n} \sum_{1}^{n} d\left(\bar{\alpha}_{j}, \bar{\beta}_{j}\right),
$$

where $\left\{\bar{\alpha}_{j}\right\}$ and $\left\{\bar{\beta}_{j}\right\}$ run over all pairs of partitions on the same space such that $\left\{\alpha_{j}\right\}_{1}^{n} \sim\left\{\bar{\alpha}_{j}\right\}_{1}^{n}$ and $\left\{\beta_{j}\right\}_{1}^{n} \sim\left\{\bar{\beta}_{j}\right\}_{1}^{n}$. Let $\alpha$ be a partition and $E$ be a subset of $M$. Then the normalized measure $\nu_{E}(A)=\nu(A \cap E) / \nu(E)$ will be associated to $\left.\alpha\right|_{E}$.

Definition 2.2. A finite partition $\alpha$ is said to be very weakly Bernoullian for $T$, if for every $\varepsilon>0$ there exists $N \geq 0$ such that for all $N^{\prime \prime}$ $\geq N^{\prime} \geq N$, all $n \geq 0$ and $\varepsilon$-a.e. $B$ in $\bigvee_{i=N^{\prime}}^{N^{\prime \prime}} T^{-i} \alpha$,

$$
\bar{d}\left(\left\{T^{i} \alpha\right\}_{1}^{n},\left\{T^{i} \alpha \mid B\right\}_{1}^{n}\right) \leq \varepsilon .
$$

D. S. Ornstein and others [2], [4], [7], [8] have shown the following theorem: 
THEOREM A. If one of the following conditions is satisfied, then $T$ is isomorphic to a Bernoulli shift.

(i) There is a weak Bernoulli generator for $T$.

(ii) There is a sequence of weak Bernoulli partitions $\alpha_{n}$ for $T$ such that $\bigvee_{i=-\infty}^{\infty} T^{i} \alpha_{n} \uparrow \epsilon^{*)}$ as $n \rightarrow \infty$.

(iii) There is a sequence of very weak Bernoulli partitions $\alpha_{n}$ for $T$ such that $\bigvee_{i=-\infty}^{\infty} T^{i} \alpha_{n} \uparrow \epsilon$ as $n \rightarrow \infty$.

In order to apply this theorem to a perturbed billiard system, it is convenient to prepare the following lemmas.

LEMMA 2.3. (i) If for any $\varepsilon>0$ and $\delta>0$, there exist a natural number $N$ and a finite family $\mathscr{F}$ of disjoint subsets of $M$ with $\sum_{F \in \mathscr{F}} \nu(F)$ $\geq 1-\delta$ such that for all $N^{\prime \prime} \geq N^{\prime} \geq N$, all $n \geq 0$ and $\varepsilon$-a.e. $B$ in $\bigvee_{i=N^{\prime}}^{N^{\prime \prime}} T^{-i} \alpha$,

$$
\begin{gathered}
\sum_{\substack{n \\
A \in V^{i} T^{i \alpha} \\
i=0}}|\nu(A \mid F)-\nu(A \mid F \cap B)|<\delta \quad \text { for any } F \text { in } \mathscr{F}, \\
\sum_{F \in \mathcal{F}}|\nu(F)-\nu(F \mid B)|<\delta,
\end{gathered}
$$

then the partition $\alpha$ is weakly Bernoullian for $T$.

(ii) In (i), the condition (2.4) is unnecessary if $T$ is a K-system and the entropy of $\alpha$ is finite.

(iii) In (i), (2.3) is fulfilled if for all $A$ in $\bigvee_{i=0}^{n} T^{i} \alpha$, all $F$ in $\mathscr{F}$ and в-a.e. $B$ in $\bigvee_{i=N^{\prime}}^{N^{\prime \prime}} T^{-i} \alpha$,

$$
\left|\frac{\nu(A \cap B \cap F) \nu(F)}{\nu(A \cap F) \nu(B \cap F)}-1\right|<\delta .
$$

Proof. Put $F_{0}=M-\bigcup_{F \in \mathscr{F}} F$, then one has

$$
\begin{aligned}
\nu\left(F_{0} \mid B\right) & \leq 1-\sum_{F} \nu(F \mid B) \leq 1-(1-\delta) \sum_{F} \nu(F) \\
& \leq 1-(1-\delta)^{2}
\end{aligned}
$$

by (2.4) and by $\sum \nu(F) \geq 1-\delta$. From (2.3) the estimate

$$
\begin{aligned}
\sum_{F} \sum_{A}|\nu(A \cap F)-\nu(A \cap F \mid B)| \\
\quad \leq \sum_{A, F}|\nu(A \mid F) \nu(F)-\nu(A \mid B \cap F) \nu(F \mid B)|
\end{aligned}
$$

*) The symbol $\in$ denotes the partition into the individual points. 


$$
\begin{aligned}
& \leq \sum_{A, F}|\nu(A \mid F)-\nu(A \mid B \cap F)| \nu(F)+\sum_{A, F} \nu(A \mid B \cap F)|\nu(F)-\nu(F \mid B)| \\
& \leq 2 \delta
\end{aligned}
$$

is obtained. Hence for $2 \varepsilon$-a.e. $B$ in $\bigvee_{i=N^{\prime}}^{N^{\prime \prime}} T^{-i} \alpha$,

$$
\begin{aligned}
\sum_{A} \mid \nu(A) & -\nu(A \mid B) \mid \\
& \leq \sum_{F, A}|\nu(A \cap F)-\nu(A \cap F \mid B)|+\sum_{A}\left\{\nu\left(A \cap F_{0}\right)+\nu\left(A \cap F_{0} \mid B\right)\right\} \\
& \leq 2 \delta+\delta+1-(1-\delta)^{2} \leq 5 \delta .
\end{aligned}
$$

Thus (i) is proved. If $T$ is a $K$-system, then for the given $\mathscr{F}$ there exists $N$ such that for all $N^{\prime \prime} \geq N^{\prime} \geq N$, all $F$ in $\mathscr{F}$ and $\varepsilon$-a.e. $B$ in $\bigvee_{N^{\prime}}^{N^{\prime \prime}} T^{-i} \alpha$,

$$
|\nu(F)-\nu(F \mid B)| \leq \delta \nu(F) .
$$

It is easily seen that (2.5) implies (2.3).

Q.E.D.

A mapping $\phi$ from $X$ to $Y$ is called $\varepsilon$-measure preserving if there exists a subset $E$ of $X$ with $\mu_{X}(E) \leq \varepsilon$ such that for all $A \subset X-E$

$$
\left|\frac{\mu_{Y}(\phi A)}{\mu_{X}(A)}-1\right|<\varepsilon
$$

Let $e(n)$ be the function on ordinal numbers defined by $e(0)=0, e(n)=1$ for $n \neq 0$. For a given partition $\alpha=\left\{A_{j}\right\}$, the name function of $\alpha$ is defined by $\ell(x) \equiv j$ if $x$ is in $A_{j}$. The following lemma is due to D. S. Ornstein and B. Weiss [9].

LEMMA 2.4. Let $\left\{\alpha_{i}\right\}_{1}^{n}$ be partitions of $X$ with name functions $\ell_{i}(x)$, and $\left\{\beta_{i}\right\}_{1}^{n}$ be partitions of $Y$ with name functions $m_{i}(y)$. If there is an $\varepsilon$-measure preserving mapping $\phi$ from $X$ to $Y$ such that

$$
\frac{1}{n} \sum_{1}^{n} e\left(\ell_{i}(x)-m_{i}(\phi x)\right) \leq \varepsilon
$$

holds for $x$ in $X-E$ with $\mu_{X}(E) \leq \varepsilon$, then

$$
\bar{d}\left(\left\{\alpha_{i}\right\}_{1}^{n},\left\{\beta_{i}\right\}_{1}^{n}\right) \leq 16 \varepsilon .
$$

The lemma is easily proved, but it is useful to check (2.2) for a suitable partition $\alpha$ (cf. $\S 6$ ).

\section{§3. Perturbed billiard systems}

In the previous article by one of the authors [5], a perturbed billiard system was defined as follows. Let $\bar{Q}_{\iota}, \iota=1,2, \cdots, I$ be disjoint strictly 
convex domains in a 2-dimensional torus $T$ whose boundaries $\partial Q$, are closed curves of $C^{3}$-class. Put $Q=T-\cup_{\imath} \bar{Q}_{\imath}$ and $\partial Q=\cup_{\iota} \partial Q_{\imath}$ and put $M_{0}=$ $\{(q, p) ; q \in Q, p=(\cos \omega, \sin \omega), 0 \leq \omega<2 \pi\}$. The flow $\left\{S_{t}\right\}$ on $M_{0}$ which describes the motion of a particle moving around in $Q$ with unit speed and with elastic collision at $\partial Q$ is called a Sinai billiard system in $Q$ [11], [12]; the particle moves along straight lines in the interior of $Q$ with speed one, and is reflected at $\partial Q$ according to the law "the angle of reflection is equal to the angle of incidence". Denote by $M$ the set of all unit incident vectors at $\partial Q$. Then every element $x=(q, p)$ of $M$ can be represented by coordinates $(\iota, r, \varphi)$, where $\iota$ is the number of $\partial Q_{t}$ containing $q, r$ is the arclength between $q$ and a fixed origin in $\partial Q_{\imath}$ measured along $\partial Q_{\text {}}$ clockwise, and $\varphi$ is the angle between $p$ and the inward normal of $\partial Q$, at $q$. For $x$ in $M_{0}$, put

$$
\begin{aligned}
& v(x)=\inf \left\{t \geq 0 ; S_{t} x \text { collides with } \partial Q\right\} \\
& t(x)=\sup \left\{t<0 ; S_{t} x \text { collides with } \partial Q\right\} .
\end{aligned}
$$

Then almost every point $x$ in $M_{0}$ (with respect to the measure $d q d \omega$ ) is parametrized by $(\iota, r, \varphi, v)$, where $v=v(x)$ and $(\iota, r, \varphi)$ represents the point $S_{v} x$ in $M$. One can define a transformation $T$ of $M$, which is called $a$ Sinai billiard transformation, by

$$
T x=S_{\tau(x)-0} x \quad \text { for } x \text { in } M .
$$

Then $\left\{S_{-t}\right\}$ is a Kakutani-Ambrose flow with the basic space $M$, the basic transformation $T$ and the ceiling function $\rightarrow \tau(x)$. The invariant measure $\mu$ of $\left\{S_{t}\right\}$ determined by Liouville's theorem is expressed in the form

$$
d \mu=-\mu_{0} \cos \varphi d \varphi d r d v d \iota
$$

and the corresponding invariant measure of $T$ is expressed in the form

$$
d \nu=-\nu_{0} \cos \varphi d \varphi d r d \iota
$$

with $\mu_{0}=(2 \pi|Q|)^{-1}$ and $\nu_{0}=(2|\partial Q|)^{-1}$, where $|Q|$ is the volume of $Q$ and $|\partial Q|$ is the total arclength of the boundary $\partial Q$.

Definition 3.1. A transformation $T_{*}$ of $M$ is called a perturbed billiard transformation if $T_{*}$ is expressed in the form

$$
T_{*}=T_{1} T,
$$

where $T$ is the Sinai billiard transformation and $T_{1}$ is a $C^{2}$-diffeomorphism 
of $M$ which preserves the measure $\nu$ and each $M^{(\iota)} \equiv\left\{(\iota, r, \varphi) ;(\iota, r) \in \partial Q_{\iota}\right\}$.

In [5], a special class of perturbed billiard transformations has been investigated $^{*)}$ :

(H-1) $T_{1}(\iota, r, \varphi)=(\iota, r-H(\iota, \varphi), \varphi)$, where $H(\iota, \varphi)$ is a function of $C^{2}$ class and satisfies $H(\iota,(\pi / 2))=H(\iota,(3 / 2) \pi)=0$,

$(\mathrm{H}-2)$ every $\bar{Q}$, are disjoint strictly convex domains whose boundaries are curves of $C^{3}$-class,

$(\mathrm{H}-3) \min _{\iota, \varphi}\left\{h(\iota, \varphi)+\left[\max _{r} k(\iota, r)+\left(\min _{\imath, r, \varphi^{\prime}}\left|\tau\left(\iota, r, \varphi^{\prime}\right)\right|\right)^{-1}\right]^{-1}\right\}>0$, where $h(\iota, \varphi) \equiv d H(\iota, \varphi) / d \varphi$ and $k(\iota, r)$ is the curvature of $\partial Q$, at $(\iota, r)$.

Under the above three assumptions the ergodicity and the $K$-property of the perturbed billiard transformation $T_{*}$ were shown in [5]. In order to describe the results, it is necessary to introduce notation and terminology. A connected curve $\gamma ; \varphi=\psi(r)$ in $M^{(\iota)}$ is called $K$-increasing if for $r \neq r^{\prime}$

$$
k_{\min } \leq \frac{\psi(r)-\psi\left(r^{\prime}\right)}{r-r^{\prime}} \leq K_{\max }(\iota)
$$

holds, where

$$
k_{\min } \equiv \min _{\iota, r} k(\iota, r) \quad \text { and } \quad K_{\max }(\iota) \equiv \max _{r} k(\iota, r)+\left(\min _{r, \varphi}\left|\tau\left(T_{*}^{-1}(\iota, r, \varphi)\right)\right|\right)^{-1} .
$$

A connected curve $\gamma ; \varphi=\psi(r)$ in $M^{(\iota)}$ is called $K$-decreasing, if for $r \neq r^{\prime}$

$$
K_{\min } \leq-\frac{\psi(r)-\psi\left(r^{\prime}\right)}{r-r^{\prime}} \leq K_{\max }
$$

holds, where $K_{\min } \equiv\left[\max _{\iota, \varphi} h(\iota, \varphi)+k_{\min }^{-1}\right]^{-1}$ and $K_{\max } \equiv \max _{\iota}\left[\min _{\varphi} h(\iota, \varphi)+\right.$ $\left.K_{\max }(\iota)^{-1}\right]^{-1}$. Put $S \equiv\{(\iota, r, \varphi) \in M ; \varphi=\pi / 2$ or $3 \pi / 2\}$. Then $T_{*}^{-1} S$ (resp. $\left.T_{*} S\right)$ is called the curves of discontinuity of $T_{*}$ (resp. $T_{*}^{-1}$ ). The image $T_{*}^{-1} S$ (resp. $T_{*} S$ ) consists of a countable number of $K$-increasing (resp. $K$ decreasing) curves. The curves of $T_{*}^{-1} S$ (resp. $T_{*} S$ ) decompose $M$ into connected components and define the partition $\alpha^{(e)}\left(\right.$ resp. $\left.\alpha^{(c)}\right)$ into the components $\left\{X_{j}^{(e)}\right\}$ (resp. $\left\{X_{j}^{(c)}\right\}$ ). Then $T_{*}$ (resp. $T_{*}^{-1}$ ) is continuous in the interior of each component and belongs to $C^{2}$-class. If $T_{*}$ (resp. $T_{*}^{-1}$ ) is continuous on a connected $K$-decreasing (resp. $K$-increasing) curve $\gamma$, then so is the image of $\gamma$.

For a point $x=(\iota, r, \varphi)$ in $M$, put $x_{i}=\left(\iota_{i}, r_{i}, \varphi_{i}\right) \equiv T_{*}^{-i}(\iota, r, \varphi),\left(\iota_{i}, r_{i}^{\prime}, \varphi_{i}^{\prime}\right)$ $\equiv T_{1}^{-1} x_{i}, k_{i} \equiv k\left(\iota_{i}, r_{i}\right), k_{i}^{\prime} \equiv k\left(\iota_{i}, r_{i}^{\prime}\right), h_{i} \equiv h\left(\iota_{i}, \varphi_{i}\right)$ and $\tau_{i} \equiv \tau\left(\iota_{i}, r_{i}, \varphi_{i}\right)$. Define

\footnotetext{
*) A more general case was discussed in [6].
} 
functions $b_{n}(x ; t),-\infty<n<\infty$, of $(x, t)$ in $M \times(-\infty, \infty)$ by

$$
\begin{aligned}
& b_{1}(x ; t) \equiv \frac{\left(\cos \varphi+k^{\prime} \tau_{1}\right)(h+t)+\tau_{1}}{\left(k_{1} \cos \varphi+k^{\prime} \cos \varphi_{1}+k_{1} k^{\prime} \tau_{1}\right)(h+t)+\cos \varphi_{1}+k_{1} \tau_{1}} \\
& b_{n+1}(x ; t) \equiv b_{1}\left(T_{*}^{-n} x ; b_{n}(x ; t)\right) \quad n \geq 1 \\
& b_{0}(x ; t) \equiv t \\
& b_{-1}(x ; t) \equiv-h-\frac{(\cos \varphi+k \tau) t-\tau}{\left(k \cos \varphi_{-1}+k_{-1}^{\prime} \cos \varphi+k k_{-1}^{\prime} \tau\right) t-\left(\cos \varphi_{-1}+k_{-1}^{\prime} \tau\right)} \\
& b_{-n-1}(x ; t) \equiv b_{-1}\left(T_{*}^{n} x ; b_{-n}(x ; t)\right) \quad n \geq 1 .
\end{aligned}
$$

Suppose that $\gamma$ and $T_{*}^{-n} \gamma$ are given by the equations $r=u(\varphi)$ and $r_{n}$ $=u_{n}\left(\varphi_{n}\right)$, respectively, with $T_{*}^{-n}(\iota, u(\varphi), \varphi)=\left(\iota_{n}, u_{n}\left(\varphi_{n}\right), \varphi_{n}\right)$. Then the formula

$$
\frac{d u_{n}\left(\varphi_{n}\right)}{d \varphi_{n}}=b_{n}\left(\iota, r, \varphi ; \frac{d u(\varphi)}{d \varphi}\right)
$$

holds for all $n$. Further one can see that for $(x, t)$ in $M \times\left[1 / K_{\min }, \infty\right)$ $b_{n}\left(T_{*}^{n} x ; t\right)$ converges to a positive function $1 / \chi^{(e)}(x)$ and that $b_{-n}\left(T_{*}^{-n} x ;-t\right)$ converges to a negative function $1 / \chi^{(c)}(x)$. The function $\chi^{(c)}$ (resp. $\chi^{(e)}$ ) is continuous at $x$ not in $\bigcup_{n=0}^{\infty} T_{*}^{n} S$ (resp. $\bigcup_{n=0}^{\infty} T_{*}^{-n} S$ ) and satisfies

$$
\left.K_{\min } \leq-\chi^{(c)}(x) \leq K_{\max } \text { (resp. } k_{\min } \leq \chi^{(e)}(x) \leq K_{\max }(c)\right)
$$

TheOREM 1. (i) $\alpha^{(c)}$ and $\alpha^{(e)}=T_{*}^{-1} \alpha^{(c)}$ are generators for $T_{*}$ with the same finite entropy.

(ii) Almost every element of $\zeta^{(c)} \equiv \bigvee_{i=0}^{\infty} T_{*}^{i} \alpha^{(c)}$ is a connected $K$ decreasing curve whose gradient ${ }^{*}$ at $x$ is equal to $\chi^{(c)}(x)$. Alternatively, almost every element of $\zeta^{(e)} \equiv \bigvee_{i=0}^{\infty} T_{*}^{-i} \alpha^{(e)}$ is a connected $K$-increasing curve whose gradient at $x$ is equal to $\chi^{(e)}(x)$.

(iii) $T_{*}$ is a K-system. Actually, the partition $\zeta^{(c)}$ and the partition $\zeta^{(e)}$ satisfy the following conditions:

$$
\begin{aligned}
& T_{*}^{-1} \zeta^{(e)}>\zeta^{(c)}, \quad T_{*} \zeta^{(e)}>\zeta^{(e)}, \\
& \bigvee_{i} T_{*}^{i} \zeta^{(c)}=\bigvee_{i} T_{*}^{i} \zeta^{(e)}=\epsilon \\
& \bigwedge_{i} T_{*}^{i} \zeta^{(c)}=\bigwedge_{i} T_{*}^{i} \zeta^{(e)}=\text { the trivial partition } .
\end{aligned}
$$

By the theorem, in order to show the Bernoulli property of $T_{*}$, it is enough to give a family $\mathscr{F}$ which satisfies the condition (2.5) in Lemma 2.3. For this purpose, it is necessary to investigate the structure of the

*) When a curve $\gamma$ is given by the equation $\varphi=\psi(r)$, the gradient of $\gamma$ at $x=(r, \varphi)$ is $d \psi / d r$. 
measure $\mu$ in connection with the partitions $\zeta^{(c)}$ and $\zeta^{(e)}$. Denote by $\gamma^{(c)}(x)$ the curve which is the element of $\zeta^{(c)}$ involving $x$. Alternatively, denote by $\gamma^{(e)}(x)$ the curve which is the element of $\zeta^{(e)}$ including $x$. For two decreasing curves $\gamma$ and $\gamma^{\prime}$, define the canonical mapping $\Psi_{\gamma^{\prime}, \gamma}^{(e)}$ by

$$
\begin{aligned}
& \Psi_{r^{\prime}, r}^{(e)}: \gamma \rightarrow \gamma^{\prime} \\
& \Psi_{r^{\prime}, r}^{(e)}: x \mapsto \gamma^{(e)}(x) \cap \gamma^{\prime} .
\end{aligned}
$$

Let $\sigma_{\gamma}$ and $\sigma_{\gamma^{\prime}}$ be the measures on $\gamma$ and $\gamma^{\prime}$ respectively defined as follows; for $\bar{\gamma}$ in $\gamma$ and $\bar{\gamma}^{\prime}$ in $\gamma$

$$
\sigma_{r}(\bar{\gamma})=\int_{\bar{\gamma}}|d \varphi| \text { and } \sigma_{\gamma^{\prime}}\left(\bar{\gamma}^{\prime}\right)=\int_{\bar{\gamma}^{\prime}}|d \varphi| .
$$

Define the measure $\Psi_{r, r}^{(e)} \sigma_{r^{\prime}}$ by

$$
\Psi_{r, r^{\prime}}^{(e)} \sigma_{\gamma^{\prime}}(\bar{\gamma}) \equiv \sigma_{r^{\prime}}\left(\Psi_{\gamma^{\prime}, r}^{(e)} \bar{\gamma}\right)
$$

Then the Radon-Nikodym density relative to $d \sigma_{r}$ is given by

$$
\frac{d \Psi_{\gamma, \gamma^{\prime}}^{(e)} \sigma_{\gamma^{\prime}}}{d \sigma_{\gamma}}=g_{r, \gamma^{\prime}}^{(e)} \equiv \prod_{i=-\infty}^{0} \frac{\Lambda^{*}\left(x_{i}, T_{*}^{-i} \gamma\right)}{\Lambda^{*}\left(x_{i}^{\prime}, T_{*}^{-i} \gamma^{\prime}\right)}
$$

with $x$ in $\gamma$ and $x^{\prime}=\Psi_{\gamma^{\prime}, r}^{(e)} x$, where

$$
\Lambda^{*}(x, \gamma)=\frac{\left\{k_{1} \cos \varphi+k^{\prime} \cos \varphi_{1}+k_{1} k^{\prime} \tau_{1}\right\} b_{1}(x ; d u / d \varphi)-k^{\prime} \tau_{1}-\cos \varphi}{\cos \varphi}
$$

Similarly, $\Psi_{\gamma^{\prime}, r}^{(c)}, \sigma_{\gamma^{\prime}}, \sigma_{\gamma}$ are defined for increasing curves $\gamma^{\prime}, \gamma$ and one has

$$
\frac{d \Psi_{r, \gamma^{\prime}}^{(c)} \sigma_{\gamma^{\prime}}}{d \sigma_{\gamma}}=g_{r, \gamma^{\prime}}^{(c)}=\prod_{i=0}^{\infty} \frac{\Lambda\left(x_{i}, T_{*}^{-i} \gamma\right)}{\Lambda\left(x_{i}^{\prime}, T_{*}^{-i} \gamma^{\prime}\right)}
$$

with $x$ in $\gamma$ and $x^{\prime}=\Psi_{\gamma^{\prime}, r}^{(c)} x$, where

$$
\Lambda(x, \gamma)=-\frac{\left\{k_{1} \cos \varphi+k^{\prime} \cos \varphi_{1}+k_{1} k^{\prime} \tau_{1}\right\}\{d u / d \varphi+h\}+k_{1} \tau_{1}+\cos \varphi_{1}}{\cos \varphi_{1}}
$$

By Lemmas 6.1, 6.1 and 7.1 in [5], for any $\delta>0$ there exist an even natural number $\ell_{0}=\ell_{0}(\delta, 1,1 / 4)$ and a positive function $\varepsilon_{0}=\varepsilon_{0}(x, \delta, 1)$ which guarantee the following property: For an $x$ not in $\bigcup_{i=-\ell_{0}}^{e_{0}} T_{*}^{i} S$, let $G$ be a $K$-quadrilateral*) (a domain which is enclosed by four curves such that a pair of opposite curves $\gamma_{b}(G), \gamma_{d}(G)$ are $K$-increasing and the other pair of opposite curves $\gamma_{a}(G), \gamma_{c}(G)$ are $K$-decreasing) in the $\varepsilon_{0}$-neighbourhood

\footnotetext{
*) The notation for $G$ and some properties of $G$ are explained in [5].
} 
$U_{s_{0}}(x)$ of $x$. Suppose that $\delta_{0} \equiv \theta\left(\gamma_{a}(G)\right)=\theta\left(\gamma_{b}(G)\right)^{*)}$ and that $T_{*}^{-\ell_{0}} G, T_{*}^{\ell_{0}} G$ are $K$-quadrilaterals. Then there exist subsets $G^{(c, \delta)}$ and $G^{(e, \delta)}$ which satisfy the four conditions;

$(\mathrm{C}-1)$ for all $x$ in $G^{(c, \delta)}$ (resp. $\left.G^{(e, \delta)}\right), \gamma^{(c)}(x) \cap G^{(c, \delta)}\left(\right.$ resp. $\gamma^{(e)}(x) \cap G^{(e, \delta)}$ ) is a connected segment which joins $\gamma_{b}(G)$ and $\gamma_{d}(G)$ (resp. $\gamma_{a}(G)$ and $\gamma_{c}(G)$ ),

(C-2) $\nu\left(G^{(c, \delta)}\right) \geq(1-\delta) \nu(G)$ and $\nu\left(G^{(e, \delta)}\right) \geq(1-\delta) \nu(G)$,

(C-3) for any $K$-increasing (resp. $K$-decreasing) curve $\gamma$ and $\gamma^{\prime}$ in $G$, the canonical mapping $\Psi_{\gamma^{\prime}, \gamma}^{(c)}$ (resp. $\Psi_{\gamma^{\prime}, r}^{(e)}$ ) is absolutely continuous on $\gamma \cap G^{(c, \delta)}$ (resp. $\gamma \cap G^{(e, \delta)}$ ) with respect to $\sigma_{r}$ and $\sigma_{r^{\prime}}$,

(C-4) for any $m \geq 0, T_{*}^{-m} G^{(c, \delta)} \cap V_{m}\left(\delta_{0}\right)=\phi^{* *)}\left(\operatorname{resp} . T_{*}^{m} G^{(e, \delta)} \cap V_{m}\left(\delta_{0}\right)=\phi\right)$.
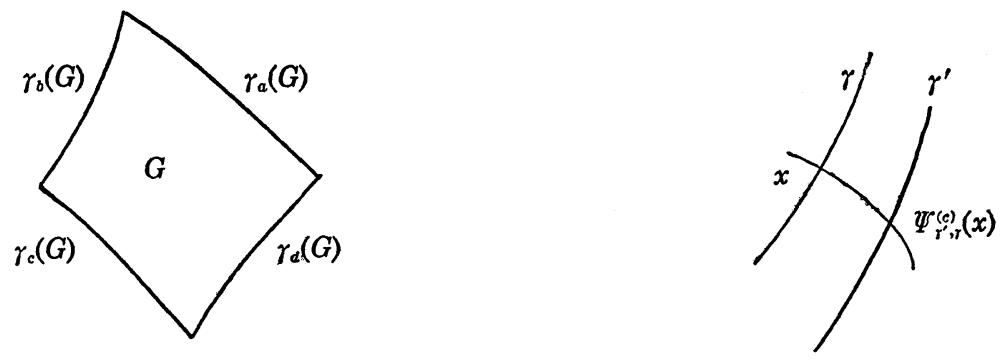

Fig. 1

Define the measure $\rho_{r}$ on a $K$-decreasing (or $K$-increasing) curve $\gamma$ by

$$
\rho_{r}(\bar{\gamma}) \equiv \int_{\bar{r}} d r \quad \text { for } \bar{\gamma} \subset \gamma
$$

Let $\gamma$ be a $K$-decreasing curve in $G$ which joins $\gamma_{a}(G)$ and $\gamma_{c}(G)$, and let $\tilde{\gamma}_{0}$ be a $K$-increasing curve which is an extension of $\tilde{\gamma}$ and is given by the equation $r=\tilde{u}(\varphi), \pi / 2 \leq \varphi \leq(3 / 2) \pi$. For given $\dot{\varphi}$ and $\dot{\psi}$, let $\tilde{\gamma}^{\dot{\varphi}, \psi}$ be the curve defined by $r=\tilde{u}(\varphi)-\tilde{u}(\dot{\varphi})-u^{\dot{\varphi}}(\dot{\psi})$, where $r=u^{\dot{\varphi}}(\dot{\psi})$ is the equation of $\gamma^{(c)}(\iota, \tilde{u}(\dot{\varphi}), \dot{\varphi})$. Then by Lemma 8.3 in [5], the measure $\nu$ is expressed in the form

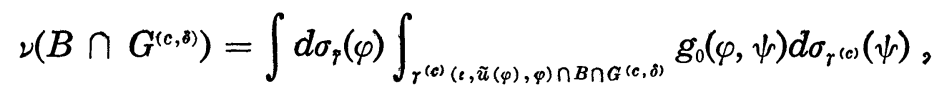

where $g_{0}(\varphi, \psi)$ is defined by

*) For monotone connected curve $\gamma, \theta(\gamma)$ denotes the total variation of $\gamma$ in $\varphi$-direction; $\theta(\gamma) \equiv \sigma_{\gamma}(\gamma)=\int_{\gamma} d \varphi$.

**) $V_{m}\left(\delta_{0}\right) \equiv\left\{x ;-\cos \varphi(x) \leq\left(1+\eta_{1}\right)^{-m / 32} \delta_{0}\right\}$ with $\eta \equiv k_{\min }|\tau|_{\min }$ and $\eta_{1} \equiv \min \{\eta,(1-r)$. $\left.K_{\min } / K_{\max }\right\}$. 


$$
g_{0}(\varphi, \psi)=\frac{\nu_{0} \cos \varphi}{\chi^{(c)}\left(\iota, u^{\varphi}(\psi), \psi\right)} \prod_{i=0}^{\infty} \frac{\Lambda\left(T_{*}^{-i} x ; T_{*}^{-i} \tilde{\gamma}_{0}\right)}{\Lambda\left(T_{*}^{-i} \hat{x} ; T_{*}^{-i} \tilde{\gamma}^{\varphi, \psi}\right)}
$$

with $x=(\iota, \tilde{u}(\varphi), \varphi)$ and $\hat{x}=\left(\iota, u^{\varphi}(\psi), \psi\right)$.

Let $\hat{\gamma}$ be a $K$-decreasing curve in $G$ which joins $\gamma_{b}(G)$ and $\gamma_{d}(G)$, and be defined by the equation $r=\hat{u}(\varphi)$. Then by (3.9), one can see that

$$
\begin{aligned}
\nu(B & \left.\cap G^{(c, \tilde{\delta})} \cap G^{(e, \tilde{\delta})}\right) \\
& =\int_{\tilde{\gamma} \cap G^{(e, \delta)}} d \sigma_{\tilde{\gamma}}(\varphi) \int_{\hat{\gamma} \cap \Psi_{\hat{\gamma}, r^{\prime}\left(B \cap G^{(c, \delta)} \cap r^{\prime}\right)}^{(e)}} g_{0}\left(\varphi, \Psi_{\hat{\gamma}, r^{\prime}}^{(e)} \hat{\psi}\right) g_{\hat{\gamma}, r^{\prime}}^{(e)}(\hat{\psi}) d \sigma_{\hat{\gamma}}(\hat{\psi})
\end{aligned}
$$

where $\gamma^{\prime}=\gamma^{(c)}(\iota, \tilde{u}(\varphi), \varphi), \Psi_{\gamma^{\prime}, r}^{(e)}(\iota, \hat{u}(\hat{\psi}), \hat{\psi})=\left(\iota, u^{\varphi}(\tilde{\varphi}), \tilde{\varphi}\right)$ and $\Psi^{(e)} \hat{\psi} \equiv \tilde{\varphi}$. One can easily see that for any fixed $m \geq \ell_{0}$, there exists a positive number $\varepsilon_{2}=\varepsilon_{2}(x, m)<\varepsilon_{0}$ such that $T_{*}^{j} U_{\varepsilon_{2}}(x) \cap V_{0}\left(2\left(1+\eta_{1}\right)^{m} \varepsilon_{2}\right)=\phi$ for $|j| \leq m$.

Lemma 3.1. Suppose that $G \subset U_{\varepsilon_{2}}(x)$ be as above and $T_{*}^{-m} \hat{\gamma}$ is a Kdecreasing curve, then

$$
\begin{aligned}
\exp [ & \left.-c_{31}\left(1+\eta_{1}\right)^{-m / 2}\right] \\
& \leq \frac{-\nu_{0}}{\nu\left(B \cap G^{(e, \delta)} \cap G^{(e, \delta)}\right)} \int_{\tilde{\gamma} \cap G^{(e, \delta)}} \cos \varphi d \sigma_{\tilde{\gamma}}(\varphi) \int_{\Psi_{\gamma, \gamma^{\prime}}^{(e)}\left(B \cap G(c, \delta) \cap \gamma^{\prime}\right)} d \rho_{\gamma}(\hat{\psi}) \\
& \leq \exp \left[c_{31}\left(1+\eta_{1}\right)^{-m / 2}\right] .
\end{aligned}
$$

In particular, if $A$ is a $\left.\zeta^{(c)}\right|_{G}$-measurable subset of $G$ and $B$ is $\left.\zeta^{(e)}\right|_{G}$-measurable subset of $G$, then

$$
\begin{aligned}
\exp [ & \left.-c_{31}\left(1+\eta_{1}\right)^{-m / 2}\right] \\
& \leq \frac{\nu_{0}}{\nu\left(A \cap B \cap G^{(c, \delta)} \cap G^{(e, \delta)}\right)} \int_{\tilde{\gamma} \cap B \cap G^{(e, \delta)}} \cos \varphi d \sigma_{\tilde{\gamma}}(\varphi) \int_{\hat{\gamma} \cap A \cap G^{(c, \delta)}} d \rho_{\hat{\gamma}}(\hat{\psi}) \\
& \leq \exp \left[c_{31}\left(1+\eta_{1}\right)^{-m / 2}\right] .
\end{aligned}
$$

In order to prove this lemma, we will prepare two lemmas.

Lemma 3.2. Let $G$ be as in Lemma 3.1. Let $\hat{\gamma}$ and $\hat{\gamma}$ be K-decreasing curves in $G$ which join $\gamma_{b}(G)$ and $\gamma_{d}(G)$. If $T_{*}^{-m} \hat{\gamma}$ and $T_{*}^{-m} \hat{\gamma}$ are $K$-decreasing, then for $\hat{x}$ in $\hat{\gamma} \cap G^{(e, \delta)}$

$$
\exp \left[-c_{32}\left(1+\eta_{1}\right)^{-m / 2}\right] \leq g_{\hat{\gamma}, \hat{\gamma}}^{(e)}(\hat{x}) \leq \exp \left[c_{32}\left(1+\eta_{1}\right)^{-m / 2}\right]
$$

holds with a positive constant $c_{32}$.

Proof. Put $\gamma \equiv \gamma^{(e)}(\hat{x}) \cap G$ and $\gamma_{j}=T_{*}^{-j} \gamma$. Put $\hat{x}_{j}=T_{*}^{-j} \hat{x}$ and $\hat{x}_{j}=$ $T_{*}^{-j} \hat{x}$ with $\hat{x}=\Psi_{\hat{\gamma}, \hat{\gamma}}^{(e)}(\hat{x})$. Since $G$ is in $U_{s_{2}}(x)$ with an $x, \min \{-\cos \varphi(y) ; y$ $\left.\in \gamma_{j}\right\} \geq 2 \varepsilon_{2}\left(1+\eta_{1}\right)^{m}$ holds for $-m \leq j \leq 0$ and $\theta(\gamma) \leq 2 \varepsilon_{2}$ holds. Put $c_{2}=$ 
$K_{\max } / K_{\min }$. Applying Lemma 5.3 and Lemma 5.4 (i) in [5], one has the estimation

$$
\log \left[\Lambda^{*}\left(\hat{x}_{j}, \hat{\gamma}_{j}\right) / \Lambda^{*}\left(\hat{x}_{j}, \hat{\gamma}_{j}\right)\right] \leq\left(c_{22}+c_{21}+\log c_{2}\right)\left(1+\eta_{1}\right)^{j-m}
$$

for $-m \leq j \leq 0$. Since $\hat{x}$ is in $G^{(e, \delta)}$, it holds that $\min \left\{-\cos \varphi(y) ; y \in \gamma_{j}\right\}$ $\geq \delta_{0}\left(1+\eta_{1}\right)^{j / 32}$ for $j \leq 0$ and that $\theta(\gamma) \leq\left(1+c_{2}\right) \delta_{0}$. Therefore again one has

$$
\log \left[\Lambda^{*}\left(\hat{x}_{j}, \hat{\gamma}_{j}\right) / \Lambda^{*}\left(\hat{x}_{j}, \hat{\gamma}_{j}\right)\right] \leq 2\left(c_{2}+1\right) c_{22}\left(1+\eta_{1}\right)^{31 j / 32}+\left(\log c_{2}\right)\left(1+\eta_{1}\right)^{j}
$$

for $j \leq-m$. These estimates imply Lemma 3.2 by (3.9).

Q.E.D.

Lemma 3.3. Let $G$ be as in Lemma 3.1 and let $T_{*}^{m} \tilde{\gamma}_{0}$ be K-increasing. Then

$$
\begin{aligned}
\exp \left[-c_{33}\left(1+\eta_{1}\right)^{-m / 2}\right] & \leq \frac{g_{0}(\varphi, \psi)}{\nu_{0}} \frac{\cos \varphi}{\cos \psi} \frac{\chi^{(c)}(\iota, u(\psi), \psi)}{\chi^{(c)}(\iota, \tilde{u}(\varphi), \varphi)} \\
& \leq \exp \left[c_{33}\left(1+\eta_{1}\right)^{-m / 2}\right]
\end{aligned}
$$

with a positive constant $c_{33}$.

Proof. Put $x=(\iota, \tilde{u}(\varphi), \varphi)$ and $\hat{x}=\left(\iota, u^{\varphi}(\psi), \psi\right)$. Similarly in Lemma 3.2,

$$
\sum_{j=0}^{\infty}\left|\log \frac{\Lambda\left(T_{*}^{-j} x, T_{*}^{-j} \tilde{\gamma}_{0}\right)}{\Lambda\left(T_{*}^{-j} \hat{x}, T_{*}^{-j} \tilde{\gamma}^{\varphi, \psi}\right)}\right| \leq c_{32}^{\prime}\left(1+\eta_{1}\right)^{-m}
$$

By Lemma 5.3 in [5], for $(\iota, \hat{u}(\hat{\varphi}), \hat{\varphi})=\Psi_{\hat{\gamma}, \gamma^{(c)}(\iota, \tilde{u}(\varphi), \varphi)}\left(\iota, u^{\varphi}(\psi), \psi\right)$

$$
\left|\log \frac{\chi^{(c)}\left(\iota, u^{\varphi}(\psi), \psi\right)}{d \hat{u} / d \hat{\psi}}\right| \leq\left(\pi c_{21}+c_{3}\right)\left(1+\eta_{1}\right)^{-m} .
$$

On the other hand, the estimate

$$
\left|\log \frac{\cos \varphi}{\cos \psi}\right| \leq 2\left(1+\eta_{1}\right)^{-m}
$$

holds, since $G$ is in $U_{\varepsilon_{2}}(x)$.

Q.E.D.

Proof of Lemma 3.1. Since $|d r|=|d \hat{u}| d \hat{\psi} \mid d \hat{\psi}$ on $\hat{\gamma}$, Lemma 3.2 and Lemma 3.3 imply the first statement in Lemma 3.1. If $A$ and $B$ are as in Lemma 3.1, then

$$
\Psi_{\hat{\gamma}, r^{\prime}}^{(e)}\left(A \cap B \cap G^{(c, \delta)} \cap \gamma^{\prime}\right)= \begin{cases}B \cap G^{(e, \delta)} \cap \hat{\gamma} & \text { if } \gamma^{\prime} \subset A \cap G^{(c, \delta)} \\ \phi & \text { if } \gamma^{\prime} \cap A \cap G^{(c, \delta)}=\phi\end{cases}
$$

where $\gamma^{\prime}=\gamma^{(c)}(y)$ with some $y$. Further, $\gamma^{\prime} \subset A \cap G^{(c, \delta)}$ if and only if $\tilde{\gamma} \cap$ $\gamma^{\prime}$ is in $\tilde{\gamma} \cap A \cap G^{(c, s)}$. Therefore one has the second statement. Q.E.D. 
§4. The perturbed billiard transformation is isomorphic to a Bernoulli shift

Applying the lemmas in $\S 2$ and $\S 3$, the following theorems will be shown.

TheOREM 2. Under the assumptions $(\mathrm{H}-1) \sim(\mathrm{H}-3), \alpha^{(c)}$ and $\alpha^{(e)}$ are weak Bernoulli generators for $T_{*}$. Thus $T_{*}$ is isomorphic to a Bernoulli shift.

Proof. By Theorem 1, it is sufficient for the proof to give a family $\mathscr{F}$ which satisfies (2.3) in Lemma 2.3. For given $\delta>0$, let $m_{0}$ be a natural number such that $\exp c_{31}\left(1+\eta_{1}\right)^{-m_{0} / 2}<1+\delta$ and $m_{0} \geq \ell_{0} \equiv \ell_{0}(1,1 / 4, \delta)$. For every $x$ not in $\bigcup_{i=-m_{0}}^{m_{0}} T^{i} S$ and for any $\delta_{0}>0$, there exists a $K$ quadrilateral $G$ in $U_{s_{2}\left(x, m_{0}\right)}(x)$ such that $\theta\left(\gamma_{a}(G)\right)=\theta\left(\gamma_{b}(G)\right)<\delta_{0}$, G involves the point $x$ and $T_{*}^{-m_{0}} G, T_{*}^{m_{0}} G$ are $K$-quadrilaterals. By the covering theorem of Vitali, there exists a finite family $\mathscr{G}$ of such $G$ 's which satisfies $G \cap G^{\prime}$ $=\phi$ for $G \neq G^{\prime}$ in $\mathscr{G}$ and $\nu\left(M-\bigcup_{G \in \mathcal{G}} G\right)<\delta$. Then by Lemmas 6.1, 6.1' and 7.1 in [5], there exist subsets $G^{(c, \delta)}$ and $G^{(e, \delta)}$ which satisfy (C-1), (C-2) and $(\mathrm{C}-3)$ in $\S 3$. Let $A$ be an element of the partition $\bigvee_{i=0}^{n} T_{*}^{i} \alpha^{(c)}$ and let $B$ be an element of $\bigvee_{i=N^{\prime}}^{N^{\prime \prime}} T_{*}^{-i} \alpha^{(c)}$. Since $A$ is $\zeta^{(c)}$-measurable and $B$ is $\zeta^{(e)}$-measurable, Lemma 3.1 is applicable. Put $\tilde{\gamma} \equiv \gamma^{(c)}(x) \cap G$ and $\hat{\gamma} \equiv \gamma^{(e)}(x)$

$\cap G$ for a fixed $x$ in $G^{(c, \delta)} \cap G^{(e, \delta)}$. Then one has

$$
\begin{aligned}
(1+\delta)^{-1} \leq \frac{-\nu_{0}}{\nu\left(A \cap B \cap G^{(c, \delta)} \cap G^{(e, \delta)}\right)} & \int_{\tilde{\gamma} \cap B \cap G(e, \delta)} \cos \varphi d \sigma(\varphi) \\
& \times \int_{\hat{\gamma} \cap A \cap G(c, \delta)} d \rho(\gamma) \leq 1+\delta .
\end{aligned}
$$

Since $A$ and $B$ are arbitrary, the above inequality holds even if one replaces $A$ to $G^{(c, \delta)}\left(B\right.$ to $\left.G^{(e, \delta)}\right)$. Hence the estimate

$$
(1+\delta)^{-4} \leq \frac{\nu\left(A \cap B \cap G^{(c, \delta)} \cap G^{(e, \delta)}\right) \nu\left(G^{(c, \delta)} \cap G^{(e, \delta)}\right)}{\nu\left(A \cap G^{(c, \delta)} \cap G^{(e, \delta)}\right) \nu\left(B \cap G^{(c, \delta)} \cap G^{(e, \delta)}\right)} \leq(1+\delta)^{4}
$$

is obtained. Therefore the family $\mathscr{F} \equiv\left\{G^{(c, \delta)} \cap G^{(e, \delta)} ; G \in \mathscr{G}\right\}$ satisfies (2.3).

Q.E.D.

Corollary 4.1. A Sinai billiard transformation is isomorphic to a Bernoulli shift. In particular, the natural generators $\alpha^{(c)}$ and $\alpha^{(e)}$ are weakly Bernoullian for $T$.

Let $\alpha=\left\{X_{j}\right\}$ be a countable partition. Denote the boundary of $X_{j}$ by $\partial X_{j}$. The union $\partial \alpha \equiv \bigcup_{j} \partial X_{j}$ is called the boundary of the partition $\alpha$. 
Let $d(x ; \partial \alpha)$ be the distance between a point $x$ in $M$ and the boundary $\partial \alpha$.

Lemma 4.2. (i) If $\log d(x ; \partial \alpha)$ is integrable, then the entropy of $\alpha$ is finite.

(ii) If the boundary $\partial \alpha$ consists of curves whose total arclength is finite, then $\log d(x ; \partial \alpha)$ is integrable.

Proof. Put $R=\sup _{x \in X_{j}} d\left(x ; \partial X_{j}\right)$, then for $x \in X_{j}$

$$
\begin{aligned}
\nu\left(X_{j}\right) & \geq-\nu_{0} \int_{0}^{R} \int_{\pi / 2}^{R+\pi / 2} \cos \varphi d \varphi d r \geq \nu_{0} R^{8} / 4 \\
& \geq \nu_{0}\left\{d\left(x ; \partial X_{j}\right)\right\}^{3} / 4 .
\end{aligned}
$$

This inequality implies $-\sum \nu\left(X_{j}\right) \log \nu\left(X_{j}\right)<\infty$. The second assertion is obvious.

Q.E.D.

THEOREM 3. Under the assumptions $(\mathrm{H}-1) \sim(\mathrm{H}-3)$, every countable partition $\alpha$ is weakly Bernoullian for $T_{*}$ whenever $\log d(x ; \partial \alpha)$ is integrable.

Proof. For a fixed $x$ and $i>0$, the distance between $T_{*}^{-i} x$ and $T_{*}^{-i} \gamma^{(c)}(x) \cap \partial \alpha$ measured along $\gamma^{(c)}\left(T_{*}^{-i} x\right)$ is greater than $d\left(T_{*}^{-i} x ; \partial \alpha\right)$, if $T_{*}^{-i} \gamma^{(c)}(x)$ intersects $\partial \alpha$. Hence

$$
d\left(T_{*}^{-i} x ; \partial \alpha\right) \leq c_{1} \theta\left(T_{*}^{-i} \gamma^{(c)}(x)\right) \leq \pi c_{1}\left(1+\eta_{1}\right)^{-i}
$$

holds with $c_{1} \equiv\left(1+K_{\min }^{-2}\right)^{1 / 2}$, if $T_{*}^{-i} \gamma^{(c)}(x)$ intersects $\partial \alpha$. Since $\log d(x ; \partial \alpha)$ is integrable, for almost every $x, 1 / i \log d\left(T_{*}^{-i} x ; \partial \alpha\right)$ converges to 0 as $i \rightarrow \infty$, by the Birkhoff ergodic theorem. Thus for almost every $x$, the boundary $\partial \alpha$ is not intersected by $T_{*}^{-i} \gamma^{(c)}(x)$ of infinitely many $i$ 's. Hence for almost every $x$, there exists a natural number $n^{(c)}(x)$ such that for all $i \geq n^{(c)}(x)$ $\gamma^{(c)}(x)$ is included in an element of $T_{*}^{i} \alpha$. Further since $\log d(x ; \partial \alpha)$ is integrable, the partition of $\gamma^{(c)}\left(T_{*}^{-i} x\right)$ into the connected components of the sets $\left\{\gamma^{(c)}\left(T_{*}^{-i} x\right) \cap X_{j}\right\}_{j=1}^{\infty}$ is a countable partition. Put

$$
\zeta_{\alpha}^{(c)} \equiv \bigvee_{i=0}^{\infty} T_{*}^{-i} \alpha \quad \text { and } \quad \zeta_{\alpha}^{(e)} \equiv \bigvee_{i=1}^{\infty} T_{*}^{i} \alpha
$$

Then by the above discussions, the restriction of the partition $\zeta_{\alpha}^{(c)}$ to almost every element $\gamma^{(c)}$ of $\zeta^{(c)}$ is a countable partition, whose elements are countable unions of connected segments of $\gamma^{(c)}$. Let $C^{(c)}(x)$ be the connected component of $x$ in the element of $\zeta_{\alpha}^{(c)} \vee \zeta^{(c)}$ which contains $x$. The partition $\zeta_{a}^{(e)}$ and $C^{(e)}(x)$ are similarly defined. Denote by $\varphi(x)$ the $\varphi$-coordinate of $x=(\iota, r, \varphi)$. Then for almost every $x$ 


$$
\begin{array}{ll}
\bar{\theta}\left(C^{(c)}(x) ; x\right) \equiv \sup _{y \in C^{(c)}(x)} \varphi(y)-\varphi(x), & \underline{\theta}\left(C^{(c)}(x) ; x\right) \equiv \varphi(x)-\inf _{y \in C^{(c)}(x)} \varphi(y) \\
\bar{\theta}\left(C^{(e)}(x) ; x\right) \equiv \sup _{y \in C^{(e)}(x)} \varphi(y)-\varphi(x), & \underline{\theta}\left(C^{(e)}(x) ; x\right) \equiv \varphi(x)-\inf _{y \in C^{(e)}(x)} \varphi(y)
\end{array}
$$

are all positive.

For $\delta>0$, let $\ell_{0}, m_{0}$ and $\varepsilon_{2}$ be as in the proof of Theorem 2. Let $t$ be a number which satisfies $\nu(E)>1-\delta$ with $E=\left\{x ; \bar{\theta}\left(C^{(c)}(x) ; x\right)>t, \underline{\theta}\left(C^{(c)}(x)\right.\right.$; $x)>t, \bar{\theta}\left(C^{(e)}(x) ; x\right)>t$ and $\left.\underline{\theta}\left(C^{(e)}(x) ; x\right)>t\right\}$. By the similar way to the proof of Theorem 2, there exists a finite family $\mathscr{G}_{1}$ of $K$-quadrilaterals such that

$$
\begin{aligned}
& \theta\left(\gamma_{a}(G)\right)=\theta\left(\gamma_{b}(G)\right)<t /\left(1+K_{\max } / K_{\min }\right), \\
& \nu(E \cap G)>(1-\delta) \nu(G), \\
& \nu\left(E-\bigcup_{G \in \mathscr{G}_{1}} G\right)<\delta, \\
& G \cap G^{\prime}=\phi \quad \text { if } G \neq G^{\prime},
\end{aligned}
$$

and that $T_{*}^{-m_{0}} G, T_{*}^{m_{0}} G$ are $K$-quadrilaterals and there exist subsets $G^{(c, \hat{o})}$ and $G^{(e, s)}$ of $G$ which satisfy the conditions $(\mathrm{C}-1),(\mathrm{C}-2),(\mathrm{C}-3)$, and $(\mathrm{C}-4)$ in $\S 3$. For $G$ in $\mathscr{G}_{1}$, put

$$
\begin{aligned}
& \hat{G}^{(c)} \equiv\left\{x \in G^{(c, \delta)} ; C^{(e)}(x) \text { intersects both } \gamma_{b}(G) \text { and } \gamma_{d}(G)\right\}, \\
& \hat{G}^{(e)} \equiv\left\{x \in G^{(e, \delta)} ; C^{(e)}(x) \text { intersects both } \gamma_{a}(G) \text { and } \gamma_{c}(G)\right\} .
\end{aligned}
$$

Then $\hat{G}^{(c)}$ is a $\left.\zeta^{(c)}\right|_{G}$-measurable subset and includes $E \cap G^{(c, \delta)}$. Alternatively, $\hat{G}^{(e)}$ is $\left.\zeta^{(e)}\right|_{G}$-measurable subset and includes $E \cap G^{(e, \delta)}$. Since for any element $A$ of $\bigvee_{0}^{n} T_{*}^{i} \alpha, A \cap \hat{G}^{(c)}$ is $\left.\zeta^{(c)}\right|_{G}$-measurable and since for any element $B$ of $\bigvee_{i=N^{\prime}}^{N^{\prime \prime}} T_{*}^{-i} \alpha, B \cap \hat{G}^{(e)}$ is $\left.\zeta^{(e)}\right|_{G}$-measurable, Lemma 3.1 is applicable to the subsets $A$ and $B$. Thus one has the estimates

$$
\begin{aligned}
& \left|\frac{\nu\left(A \cap B \cap \hat{G}^{(c)} \cap \hat{G}^{(e)}\right) \nu\left(\hat{G}^{(c)} \cap \hat{G}^{(e)}\right)}{\nu\left(A \cap \hat{G}^{(c)} \cap \hat{G}^{(e)}\right) \nu\left(B \cap \hat{G}^{(c)} \cap \hat{G}^{(e)}\right)}-1\right| \leq(1+\delta)^{4}-1, \\
& \nu\left(\hat{G}^{(c)} \cap \hat{G}^{(e)}\right) \geq \nu\left(E \cap G^{(c, \delta)} \cap G^{(e, \delta)}\right) \geq(1-3 \delta) \nu(G),
\end{aligned}
$$

and

$$
\nu\left(\bigcup_{G \in \mathscr{S}_{1}}\left(\hat{G}^{(c)} \cap \hat{G}^{(e)}\right)\right) \geq(1-2 \delta)(1-3 \delta)
$$

Hence the conditions in Lemma 2.3 are fulfilled.

Q.E.D. 


\section{§5. K-properties of the flow $\left\{S_{t}^{f}\right\}$}

Let $f(x)$ be a positive function on $M$ and let $\left\{S_{-t}^{f}\right\}$ be a KakutaniAmbrose flow with the basic space $M$, the basic transformation $T_{*}$ and the ceiling function $f(x)$; that is, $\left\{S_{t}^{f}\right\}$ is defined on the space $W \equiv\{(x, v)$; $0 \leq v<f(x), x \in M\}$ by

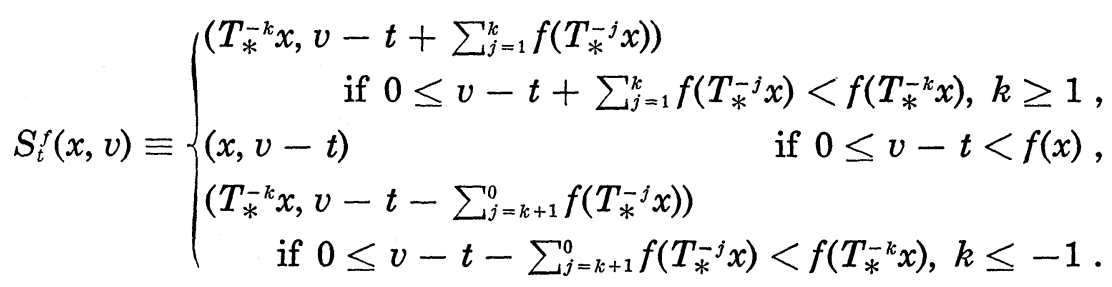

Associate the invariant probability measure $\mu_{f}$ with $\left\{S_{t}^{f}\right\}: d \mu_{f}=c_{f} d v d \nu$. Suppose that the assumptions $(\mathrm{H}-1) \sim(\mathrm{H}-3)$ are satisfied. Then $\left\{S_{t}^{f}\right\}$ is ergodic, since $T_{*}$ is ergodic. Moreover suppose the following three assumptions $(f-1) \sim(f-3)$ :

(f-1) $f(x)$ is strictly positive and continuously differentiable on each element $X_{j}^{(e)}$ of $\alpha^{(e)}$,

(f-2) there exists a constant $K$ such that

$$
\left\{\left|\frac{\partial f(\iota, r, \varphi)}{\partial r}\right|+\left|\frac{\partial f(\iota, r, \varphi)}{\partial \varphi}\right|\right\} \frac{\cos \varphi-1}{\tau} \leq K
$$

with $\left(\iota_{-1}, r_{-1}, \varphi_{-1}\right)=T_{*}(\iota, r, \varphi)$,

(f-3) $f(x) \log |\tau(x)|$ is integrable.

For $x$ not in $\bigcup_{i=0}^{\infty} T_{*}^{i} S$, put

$$
\begin{aligned}
& f^{(+)}(x) \equiv \sum_{i=1}^{\infty}\left\{\frac{1}{\chi^{(c)}\left(x_{i}\right)} \frac{\partial f\left(x_{i}\right)}{\partial r}+\frac{\partial f\left(x_{i}\right)}{\partial \varphi}\right\} \prod_{j=0}^{i-1}\left[\Lambda\left(x_{j}, \gamma^{(c)}\left(x_{j}\right)\right)\right]^{-1} \\
& \hat{f}^{(+)}(x) \equiv \sum_{i=1}^{\infty}\left\{\left|\frac{1}{\chi^{(c)}\left(x_{i}\right)} \frac{\partial f\left(x_{i}\right)}{\partial r}\right|+\left|\frac{\partial f\left(x_{i}\right)}{\partial \varphi}\right|\right\} \prod_{j=0}^{i-1}\left|\Lambda\left(x_{j}, \gamma^{(c)}\left(x_{j}\right)\right)\right|^{-1}
\end{aligned}
$$

with $x_{j} \equiv T_{*}^{-j} x$. For $x$ not in $\bigcup_{i=0}^{\infty} T_{*}^{-i} S$, put

$$
\begin{aligned}
f^{(-)}(x) \equiv & \sum_{i=-\infty}^{-1}\left\{\frac{1}{\chi^{(e)}\left(x_{i}\right)} \frac{\partial f}{\partial r}\left(x_{i}\right)+\frac{\partial f}{\partial \varphi}\left(x_{i}\right)\right\} \prod_{j=i+1}^{0}\left[\Lambda^{*}\left(x_{j}, \gamma^{(e)}\left(x_{j}\right)\right)\right]^{-1} \\
& +\frac{1}{\chi^{(e)}(x)} \frac{\partial f}{\partial r}(x)+\frac{\partial f}{\partial \varphi}(x) \\
\hat{f}^{(-)}(x) \equiv & \sum_{i=-\infty}^{-1}\left\{\left|\frac{1}{\chi^{(e)}\left(x_{i}\right)} \frac{\partial f}{\partial r}\left(x_{i}\right)\right|+\left|\frac{\partial f}{\partial \varphi}\left(x_{i}\right)\right|\right\} \prod_{j=i+1}^{0}\left|\Lambda^{*}\left(x_{j}, \gamma^{(e)}\left(x_{j}\right)\right)\right|^{-1} \\
& +\left|\frac{1}{\chi^{(e)}(x)} \frac{\partial f}{\partial r}(x)\right|+\left|\frac{\partial f}{\partial r}(x)\right| .
\end{aligned}
$$


Then by assumptions ( $\mathrm{f}-1)$, (f-2) and by Lemma 3.2, Lemma 3.3, the series in (5.2) and (5.3) converge and $f^{(+)}(x)$ (resp. $f^{(-)}(x)$ ) is continuous at $x$ not in $\bigcup_{i=0}^{\infty} T_{*}^{i} S$ (resp. $\left.\bigcup_{i=0}^{\infty} T_{*}^{-i} S\right)$.

For $w=(\tilde{x}, \tilde{v})$ in $W$, define curves $\bar{\gamma}^{(+)}(w)$ and $\bar{\gamma}^{(-)}(w)$ passing through $(\tilde{x}, \tilde{v})$ by the following way. Let $r=u^{(c)}(\varphi ; \tilde{x})$ be the equation of the curve $\gamma^{(e)}(\tilde{x})$ and let $r=u^{(e)}(\varphi ; \tilde{x})$ be the equation of the curve $\gamma^{(e)}(\tilde{x})$. Let $\bar{\gamma}^{(+)}(w)$ and $\bar{\gamma}^{(-)}(w)$ be the curves defined respectively by the equations

$$
\left\{\begin{array} { l } 
{ \iota = \tilde { \iota } } \\
{ r = u ^ { ( c ) } ( \varphi ; \tilde { x } ) } \\
{ v = \tilde { v } - \int _ { \tilde { \varphi } } ^ { \varphi } f ^ { ( - ) } ( \tilde { \iota } , u ^ { ( \iota ) } ( \varphi ; \tilde { x } ) , \varphi ) d \varphi }
\end{array} \quad \text { and } \left\{\begin{array}{l}
\iota=\tilde{\iota} \\
r=u^{(e)}(\varphi ; \tilde{x}) \\
v=\tilde{v}+\int_{\tilde{\varphi}}^{\varphi} f^{(+)}\left(\tilde{\iota}, u^{(e)}(\varphi ; \tilde{x}), \varphi\right) d \varphi
\end{array}\right.\right.
$$

for $0 \leq v<f(\iota, r, \varphi)$ with $\tilde{x}=(\tilde{\iota}, \tilde{r}, \tilde{\varphi})$. Then, obviously, $\bar{\gamma}^{(+)}(w)$ and $\bar{\gamma}^{(-)}(w)$ are locally transversal fibres; that is,

(i) for $w^{\prime}$ in $\bar{\gamma}^{(+)}(w)$ (resp. $\left.\bar{\gamma}^{(-)}(w)\right), \bar{\gamma}^{(+)}\left(w^{\prime}\right)=\bar{\gamma}^{(+)}(w)$ (resp. $\bar{\gamma}^{(-)}\left(w^{\prime}\right)=$ $\left.\bar{\gamma}^{(-)}(w)\right)$

(ii) $S_{t}^{f} \bar{\gamma}^{(+)}(w)$ coincides with $\bar{\gamma}^{(+)}\left(S_{t}^{f} w\right)$ and $S_{t}^{f} \bar{\gamma}^{(-)}(w)$ coincides with $\bar{\gamma}^{(-)}\left(S_{t}^{f} w\right)$ in a neighbourhood of $S_{t}^{f} w$.

Therefore $\tilde{\Gamma}^{(+)}(w) \equiv \bigcup_{t} S_{t}^{f} \bar{\gamma}^{(+)}\left(S_{-t}^{f} w\right)$ and $\tilde{\Gamma}^{(-)}(w) \equiv \bigcup_{t} S_{t}^{f} \bar{\gamma}^{(-)}\left(S_{-t}^{f} w\right)$ consist of countably many connected curves in $W$. Further $\tilde{\Gamma}^{(+)}(w)$ and $\tilde{\Gamma}^{(-)}(w)$ are transversal fibres; that is,

(i) $\tilde{\Gamma}^{(+)}\left(w^{\prime}\right)=\tilde{\Gamma}^{(+)}(w)$ for $w^{\prime} \in \tilde{\Gamma}^{(+)}(w)$, $\tilde{\Gamma}^{(-)}\left(w^{\prime}\right)=\tilde{\Gamma}^{(-)}(w)$ for $w^{\prime} \in \tilde{\Gamma}^{(-)}(w)$,

(ii) $S_{t}^{f} \tilde{\Gamma}^{(+)}(w)=\tilde{\Gamma}^{(+)}\left(S_{t}^{f} w\right)$ and $S_{t}^{f} \tilde{\Gamma}^{(-)}(w)=\tilde{\Gamma}^{(-)}\left(S_{t}^{f} w\right)$.

For each $x$ in $M$, identify two points $(x, f(x))$ and $\left(T_{*} x, 0\right)$. Under the identification, let $\tilde{\gamma}^{(+)}(w)$ be the connected component of $w$ in $\tilde{\Gamma}^{(+)}(w)$. Then $\left\{\tilde{\gamma}^{(+)}(w) ; w \in W\right\}$ gives a partition $\tilde{\zeta}^{(+)}$of $W$. Similarly, $\tilde{\gamma}^{(-)}(w)$ and $\tilde{\zeta}^{(-)}$are given by $\left\{\tilde{\Gamma}^{(-)}(w)\right\}$.

A curve $\tilde{\gamma}$ in $W$ which is given by the equations $\iota=\tilde{\imath}, r=u(\varphi)$ and $v=t(\varphi)$ is said to be $K$-increasing (resp. $K$-decreasing), if the curve $\gamma$ in $M^{(\bar{c})}$ defined by $r=u(\varphi)$ is $K$-increasing (resp. $K$-decreasing) and $t(\varphi)$ is locally Lipschitzc ontinuous. For a given $K$-increasing curve $\tilde{\gamma}$ in $W$, define a measure $\sigma_{\tilde{\gamma}}$ by

$$
\sigma_{\tilde{\gamma}}(A)=\int_{A}|d \varphi|
$$

for $A$ in $\tilde{\gamma}$. Put for a subset $R$ of $(-\infty, \infty)$ 


$$
\begin{aligned}
& A^{(+)}[\tilde{\gamma} ; t] \equiv \bigcup_{w \in S-t \tilde{\gamma}} \bar{\gamma}^{(+)}(w), \\
& A^{(+)}[\tilde{\gamma} ; R] \equiv \bigcup_{t \in R} A^{(+)}[\tilde{\gamma} ; t] .
\end{aligned}
$$

Let $\Pi$ be the natural projection from $W$ to $M ; \Pi(\tilde{x}, \tilde{v})=\tilde{x}$. Then for sufficiently small $R$ the measure $\mu=\mu_{f}$ satisfies

$$
\mu\left(B \cap A^{(+)}[\tilde{\gamma} ; R]\right)=c_{f} \int_{R} \nu\left(\Pi\left(A^{(+)}[\tilde{\gamma} ; t] \cap B\right)\right) d t .
$$

Similarly, subsets $A^{(-)}[\tilde{\gamma} ; t]$ and $A^{(-)}[\tilde{\gamma} ; R]$ are defined. The local fibres $\left\{\tilde{\gamma}^{(+)}\right\}$ and $\left\{\tilde{\gamma}^{(-)}\right\}$are called mutually integrable (with each other), if for almost every $w=(\tilde{x}, \tilde{v})$ in $W$ and for almost every $y$ in $\Pi\left(A^{(+)}\left[\tilde{\gamma}^{(-)}(w) ; 0\right]\right) \cap$ $\Pi\left(A^{(-)}\left[\tilde{\gamma}^{(+)}(w) ; 0\right]\right)$, the relation

$$
A^{(+)}\left[\tilde{\gamma}^{(-)}(w) ; 0\right] \cap \Pi^{-1}(y)=A^{(-)}\left[\tilde{\gamma}^{(+)}(w) ; 0\right] \cap \Pi^{-1}(y)
$$

holds.

THEOREM 4. Under the assumptions (H-1) ( $\mathrm{H}-3)$ and $(\mathrm{f}-1) \sim(\mathrm{f}-3)$,

$$
\text { (i) } \begin{array}{ll} 
& S_{t}^{f} \tilde{\zeta}^{(+)}>\tilde{\zeta}^{(+)}, S_{t}^{f} \tilde{\zeta}^{(-)}<\tilde{\zeta}^{(-)}, t>0, \\
& \bigvee_{t} S_{t}^{f} \tilde{\zeta}^{(+)}=\bigvee_{t} S_{t}^{f \tilde{\zeta}^{(-)}}=\epsilon, \\
& \bigwedge_{t} S_{t}^{f} \tilde{\zeta}^{(+)}=\bigwedge_{t} S_{t}^{f} \tilde{\zeta}^{(-)}=\pi\left(\left\{S_{t}^{f}\right\}\right),
\end{array}
$$

(ii) the conditional measure $\mu\left(\cdot \mid \tilde{\gamma}^{(+)}\right)$(resp. $\mu\left(\cdot \mid \tilde{\gamma}^{(-)}\right)$is equivalent to $\sigma_{\tilde{\gamma}^{(+)}}\left(\right.$resp. $\left.\sigma_{\tilde{\gamma}^{(-)}}\right)$,

(iii) $h\left(S_{t}^{f}\right)=h\left(S_{t}^{f} \tilde{\zeta}^{(+)} \mid \tilde{\zeta}^{(+)}\right)=h\left(S_{-t}^{f} \tilde{\zeta}^{(-)} \mid \tilde{\zeta}^{(-)}\right)=t h\left(T_{*}\right) / \int f(x) d \nu$,

(iv) if $\left\{\tilde{\gamma}^{(+)}\right\}$and $\left\{\tilde{\gamma}^{(-)}\right\}$are not mutually integrable, then $\pi\left(\left\{S_{t}^{f}\right\}\right)$ is the trivial partition, and hence $\left\{S_{t}^{f}\right\}$ is a K-system,

(v) if $\left\{S_{t}^{f}\right\}$ has no point spectrum, then $\left\{S_{t}^{f}\right\}$ is a K-system.

Proof. By the above discussions and the definitions,

$$
S_{t}^{f} \tilde{\zeta}^{(+)}>\tilde{\zeta}^{(+)}, S_{t}^{f} \tilde{\zeta}^{(-)}<\tilde{\zeta}^{(-)}(t>0) \quad \text { and } \bigvee_{t} S_{t}^{f} \tilde{\zeta}^{(+)}=\bigvee_{t} S_{t}^{f \tilde{\zeta}^{(-)}}=\epsilon
$$

are obvious. Let $\beta$ be the partition of $W$ given by $\beta \equiv \Pi^{-1} \alpha^{(e)}=\left\{\Pi^{-1} X_{j}^{(e)}\right.$; $\left.X_{j}^{(e)} \in \alpha^{(e)}\right\}$. For any countable partition $\alpha=\left\{Y_{j}\right\}$ of $W$ let $\tilde{d}(w ; \partial \alpha)$ be the distance between $w$ and the boundaries $\cup_{j} \partial Y_{j} \cup W_{*} \cup W^{*} \cup \Pi^{-1}(S)$ where $W_{*} \equiv\{(x, 0) ; x \in M\}, W^{*} \equiv\{(x, f(x)) ; x \in M\}$ and $\Pi^{-1}(S) \equiv\{(x, v) ; 0 \leq v<$ $f(x), x \in S\}$. Then $\log \tilde{d}(w ; \partial \beta)$ is integrable by virtue of $(f-1) \sim(f-3)$. Since the flow $\left\{S_{t}^{f}\right\}$ is ergodic, except for a countable number of $t$ 's the transformation $S_{t}^{f}$ is ergodic. Fix such a sufficiently small positive $t$ and suppose that $\log \tilde{d}(w ; \partial \alpha)$ is integrable. Then by the same way as in the proof 
of Theorem 3, one can see that for almost every element $\tilde{\gamma}^{(+)}$of $\tilde{\zeta}^{(+)}$, the restriction of $\zeta_{\alpha}^{(+)} \equiv \bigvee_{k=0}^{\infty} S_{-k t}^{f} \alpha$ to $\tilde{\gamma}^{(+)}$is a countable partition, each element of which is a union of a countable number of segments of $\tilde{\gamma}^{(+)}$. Hence one can see

$$
\bigwedge_{n} \bigvee_{k=0}^{\infty} S_{(n-k) t}^{f} \alpha \leq \bigwedge_{s} S_{s}^{f} \tilde{\zeta}^{(+)} \equiv \tilde{\zeta}_{\infty}^{(+)}
$$

Since there exists a sequence of partitions $\left\{\alpha_{n}\right\}$ of $W$ increasing to $\epsilon$ such that $\log \tilde{d}\left(w ; \partial \alpha_{n}\right)$ are integrable, $\pi\left(S_{t}^{f}\right) \leq \tilde{\zeta}_{\infty}^{(+)}$. If $\alpha \geq \beta$, then $\zeta_{\alpha}^{(+)} \geq \Pi^{-1} \zeta^{(c)}$ $\equiv\left\{\Pi^{-1} \gamma^{(c)} ; \gamma^{(c)} \in \zeta^{(c)}\right\}$, since $\alpha^{(c)}$ generates $\zeta^{(c)}$. For any $\varepsilon>0$ and for almost every $w,\left\{S_{-k t}^{f} w ; k \geq 0\right\}$ visits the set $Y_{c}=\{(x, v) \in W ; u-\varepsilon<v<u,(x, u)$ $\left.\in \bigcup_{j} \partial Y_{j}\right\}$ infinitely many times, since $S_{t}^{f}$ is ergodic. Hence $\zeta_{\alpha}^{(+)}=\bigvee_{k=0}^{\infty} S_{-k t}^{f} \alpha$ $\geq \tilde{\zeta}^{(+)}$if $\alpha \geq \beta$ and if $\log \tilde{d}(w ; \partial \alpha)$ is integrable. Hence one obtains

$$
\pi\left(S_{t}^{f}\right)=\pi\left(\left\{S_{s}^{f}\right\}\right)=\bigwedge_{s} S_{s}^{f} \tilde{\zeta}^{(+)}
$$

Thus (i) is proved. The second assertion (ii) is obvious by definition and $\S 3$. The third assertion (iii) comes from the theorem of Rohlin and Sinai [10] and a theorem of Abramov [1]. For almost every $y$ in $M$ and for a sufficiently small neighbourhood $U_{s}(y)$, there exists a quartet $\left\{y, y_{1}, y_{2}, y_{3}\right\}$ in $U_{\varepsilon}(y)$ such that $y_{1}$ in $\gamma^{(c)}(y), y_{2}$ in $\gamma^{(e)}\left(y_{1}\right), y_{3}$ in $\gamma^{(e)}\left(y_{2}\right)$ and $y$ in $\gamma^{(e)}\left(y_{3}\right)$. Then one can define a mapping $\Psi$ of $\Pi^{-1}(y)$ by

$$
\Psi w=\bar{\gamma}^{(-)}\left(\bar{\gamma}^{(+)}\left(\bar{\gamma}^{(-)}\left(\bar{\gamma}^{(+)}(w) \cap \Pi^{-1}\left(y_{1}\right)\right) \cap \Pi^{-1}\left(y_{2}\right)\right) \cap \Pi^{-1}\left(y_{3}\right)\right) \cap \Pi^{-1}(y)
$$

for $w$ in $\Pi^{-1}(y)$. Obviously, there exists a real number $a=a\left(y, y_{1}, y_{2}, y_{3}\right)$ such that

$$
\Psi(y, u)=(y, u+a)
$$

for $(y, u)$ in the domain of $\Psi$. If $\left\{\tilde{\gamma}^{(+)}\right\}$and $\left\{\tilde{\gamma}^{(-)}\right\}$are not mutually integrable, then there exists a subset $Y$ of positive measure such that for all $\delta>0$ and all $y$ in $Y$ one can choose a quartet $\left\{y, y_{1}, y_{2}, y_{3}\right\}$ with $0<$ $\left|a\left(y, y_{1}, y_{2}, y_{3}\right)\right|<\delta$. Put $\tilde{\zeta}_{-\infty}^{(-)} \equiv \wedge S_{t}^{f \tilde{\zeta}^{(-)}}$and let $h(w)$ be a $\tilde{\zeta}_{\infty}^{(+)} \wedge \tilde{\zeta}_{-\infty}^{(-)}$ measurable bounded function. Since $\tilde{\zeta}_{\infty}^{(+)} \wedge \tilde{\zeta}_{-\infty}^{(-)}$is $\left\{S_{t}^{f}\right\}$-invariant, $h_{b}(w)=$ $\frac{1}{b} \int_{0}^{b} h\left(S_{t}^{f} w\right) d t$ is again $\tilde{\zeta}_{\infty}^{(+)} \wedge \tilde{\zeta}_{-\infty}^{(-)}$-measurable. Then $h_{b}(y, u)$ is continuous in $u$ and $h_{b}(w)$ converges to $h(w)$ a.e. $w$ as $b \rightarrow 0$. There exist measurable functions $h_{b}^{(+)}(w)$ and $h_{b}^{(-)}(w)$ such that $h_{b}(w)=h_{b}^{(+)}(w)=h_{b}^{(-)}(w)$ for a.e. $w$ and that $h_{b}^{(+)}(w)$ is constant on $\tilde{\Gamma}^{(+)}$and $h_{b}^{(-)}(w)$ is constant on $\tilde{\Gamma}^{(-)}$. Since canonical mappings $\Psi^{(c)}$ and $\Psi^{(e)}$ are absolutely continuous, one can choose 
$y, y_{1}, y_{2}, y_{3}$ such that $h_{b}\left(y_{i}, u\right)=h_{b}^{(+)}\left(y_{i}, u\right)=h_{b}^{(-)}\left(y_{i}, u\right)$ for almost every $u$ in $\left[0, f\left(y_{i}\right)\right), i=0,1,2,3$ with $y_{0}=y$. Hence one can obtain

$$
h_{b}(y, u)=h_{b}(y, u+a)
$$

for almost every $u$ in $[\delta, f(y)-\delta]$ with small $\delta>0$. Since $\delta$ can be taken arbitrary small and $h_{b}(y, u)$ is continuous in $u, h_{b}(y, u)$ is constant in $u$. Hence $h_{b}(y, u)$ is constant in a subset with positive measure. Since $b$ and $h$ are arbitrary, one can see that the partition $\tilde{\zeta}_{\infty}^{(+)} \wedge \tilde{\zeta}_{-\infty}^{(-)}$contains an element of positive measure. Since $\left\{S_{t}^{\jmath}\right\}$ is ergodic and $\tilde{\zeta}_{\infty}^{(+)} \wedge \tilde{\zeta}_{-\infty}^{(-)}$is invariant under $\left\{S_{t}^{f}\right\}$, the partition $\zeta_{\infty}^{(+)} \wedge \zeta_{\infty}^{(-)}$is trivial. Thus (iv) was proved. Suppose that $\left\{\tilde{\gamma}^{(+)}\right\}$and $\left\{\tilde{\gamma}^{(-)}\right\}$are mutually integrable and that $\tilde{\zeta}_{\infty}^{(+)} \wedge \tilde{\zeta}_{-\infty}^{(-)}$is not trivial. Since $\wedge_{k} T_{*}^{k} \zeta^{(c)} \wedge \wedge_{k} T_{*}^{k} \zeta_{e}^{()}$is the trivial partition, the factor flow of $\left\{S_{t}^{f}\right\}$ with respect to $\tilde{\zeta}_{\infty}^{(+)} \wedge \tilde{\zeta}_{-\infty}^{(-)}$is a circle flow. Hence $\left\{S_{t}^{f}\right\}$ has a point spectrum.

Q.E.D.

It is very difficult to check that $\left\{\tilde{\gamma}^{(+)}\right\}$and $\left\{\tilde{\gamma}^{(-)}\right\}$are not mutually integrable for general cases.

Lemma 5.1. For a K-quadrilateral $G$, put

$$
v_{f}(G) \equiv \int_{r_{a}} f^{(+)} d \varphi+\int_{r_{b}} f^{(-)} d \varphi-\int_{r_{c}} f^{(+)} d \varphi-\int_{r_{d}} f^{(-)} d \varphi
$$

(i) If $v_{f}(G)=0$ for any $G$ whose lateral sides are segments of $\left\{\gamma^{(c)}\right\}$ and $\left\{\gamma^{(e)}\right\}$, then $\left\{\tilde{\gamma}^{(+)}\right\}$and $\left\{\tilde{\gamma}^{(-)}\right\}$are mutually integrable.

(ii) If $v_{f}(G)>0$ for any $G$ in an open set whose lateral sides are segments of $\left\{\gamma^{(c)}\right\}$ and $\left\{\gamma^{(e)}\right\}$, then $\left\{\tilde{\gamma}^{(+)}\right\}$and $\left\{\tilde{\gamma}^{(-)}\right\}$are not mutually integrable.

Corollary 5.2 ([12]). A Sinai billiard system is a K-system.

Proof. Since $f(x)=-\tau(x)$, it holds that

$$
\frac{1}{\chi^{(c)}(x)} \frac{\partial f}{\partial r}+\frac{\partial f}{\partial \varphi}=\frac{1}{\chi^{(c)}(x)} \sin \varphi(x)-\frac{1}{\chi^{(c)}\left(x_{1}\right)} \sin \varphi\left(x_{1}\right) \frac{d \varphi_{1}}{d \varphi},
$$

and hence $f^{(+)}(x)=\sin \varphi / \chi^{(c)}(x)$ (cf. [5]). Similarly one has $f^{(-)}(x)=$ $-\sin \varphi / \chi^{(e)}(x)$. Hence

$$
v_{f}(G)=-\int_{r_{a}} \sin \varphi d r-\int_{r_{b}} \sin \varphi d r+\int_{r_{c}} \sin \varphi d r+\int_{r_{d}} \sin \varphi d r=\frac{1}{\nu_{0}} \nu(G) .
$$

Q.E.D.

Corollary 5.3. The flow $\left\{S_{t}^{f}\right\}$ in $\S 3$ has expanding and contracting 
transversal fibres. Further if $\left\{S_{t}^{f}\right\}$ has no point spectrum, then $\left\{S_{t}^{f}\right\}$ is a K-system.

\section{§.6. Bernoulli flow}

$A$ flow $\left\{S_{t}\right\}$ is called a Bernoulli flow if every $S_{t}(t \neq 0)$ is a Bernoulli shift.

THEOREM 5. Under the assumptions $(\mathrm{H}-1) \sim(\mathrm{H}-3)$ and $(\mathrm{f}-1) \sim(\mathrm{f}-3)$, if $\left\{S_{t}^{f}\right\}$ is a K-system and $\alpha$ is a finite partition such that $\log \tilde{d}(w ; \partial \alpha)$ is integrable, then $\alpha$ is very weakly Bernoullian for $S_{t}^{f}(t \neq 0)$. Furthermore $\left\{S_{t}^{f}\right\}$ is a Bernoulli flow.

Proof. For $\varepsilon>0$, choose sufficiently small $\delta>0$. Let $\delta_{1}$ be a positive number with $\mu\left(E_{1}\right)>1-\delta$ where $E_{1} \equiv\left\{w \in W ; \tilde{d}(w ; \partial \alpha)>\delta_{1}\right\}$. Fix a positive $t$. Then $S_{t}^{f}$ is an ergodic transformation, since $\left\{S_{t}^{f}\right\}$ is a $K$-system by the assumption. Hence by Birkhoff's ergodic theorem, there exist a set $E_{2}$ with $\mu\left(E_{2}\right)>1-\delta$ and a natural number $N_{1}$ such that for all $w$ in $E_{2}$ and all $n \geq N_{1}$

$$
\frac{1}{n} \sum_{k=0}^{n-1} I_{E_{1}^{c}}\left(S_{-k t}^{f} w\right) \leq 2 \delta
$$

where $I_{E_{1}^{c}}$ is the indicator function of $E_{1}^{c}$. Then there exists a $\delta_{2}$ with $\mu\left(E_{3}\right)>1-\delta$, where $E_{3} \equiv\left\{w \in W ; \inf _{0 \leq k \leq N_{1}-1} \tilde{d}\left(S_{-k t}^{f} w ; \partial \alpha\right)>\delta_{2}\right\}$. Denote by $C^{(+)}(w)$ and $C^{(-)}(w)$ the connected components of $w$ in the elements of $\bar{\zeta}^{(+)}$ $\vee \zeta_{\alpha}^{(+)}$and $\bar{\zeta}^{(-)} \vee \zeta_{\alpha}^{(-)}$respectively, where $\zeta_{\alpha}^{(+)}=\bigvee_{k=0}^{\infty} S_{-k t}^{f} \alpha, \zeta_{\alpha}^{(-)}=\bigvee_{k=0}^{\infty} S_{k t}^{f} \alpha$ and $\bar{\zeta}^{(+)}$(resp. $\bar{\zeta}^{(-)}$) is the partition into curves $\left\{\bar{\gamma}^{(+)}\right\}$(resp. $\left\{\bar{\gamma}^{(-)}\right\}$). By the same reason in the proof of Theorem 3, there exists a positive number $\delta_{3}$ such that $\mu\left(E_{4}\right)>1-\delta$ where $E_{4} \equiv\left\{w \in W ; \bar{\theta}\left(\Pi\left(C^{(+)}(w)\right) ; \Pi(w)\right)>\delta_{3}\right.$, $\underline{\theta}\left(\Pi\left(C^{(+)}(w)\right) ; \Pi(w)\right)>\delta_{3}, \bar{\theta}\left(\Pi\left(C^{(-)}(w)\right) ; \Pi(w)\right)>\delta_{3}$ and $\underline{\theta}\left(\Pi\left(C^{(-)}(w)\right) ; \Pi(w)\right)>$ $\left.\delta_{3}\right\}$. There exists a positive $\delta_{4}(<\delta)$ such that $1 / \delta_{4}>\sup _{x \in E_{5}}\left\{\left|f^{(+)}(x)\right|+\right.$ $\left.\left|f^{(-)}(x)\right|\right\}$ with $E_{5} \equiv\left\{x \in M ;|\cos \varphi(x)|>\delta_{1}\right\}$. Note that $\Pi^{-1}\left(E_{5}\right)$ is a subset of $E_{1}$.

For any $x$ not in $\bigcup_{i=-\infty}^{\infty} T_{*}^{i} S$, there exists $\varepsilon_{3}=\varepsilon_{3}(x)>0$ such that for any $y$ in $\varepsilon_{3}$-neighbourhood $U_{\varepsilon_{3}}(x)$

$$
\begin{aligned}
& \left|f^{(+)}(x)-f^{(+)}(y)\right|<\delta_{4} \delta,\left|f^{(-)}(x)-f^{(-)}(y)\right|<\delta_{4} \delta \\
& |f(x)-f(y)|<\delta_{2} \delta \quad \text { and }\left|\frac{\cos \varphi(y)}{\cos \varphi(x)}\right|<1+\delta .
\end{aligned}
$$

Let $\ell_{0}=\ell_{0}(\delta, 1,1 / 4), m_{0} \geq \ell_{0}$ and $\varepsilon_{2}=\varepsilon_{2}\left(x, m_{0}\right)$ be as in the proof of Theorem 
2. Then for any $w=(\tilde{\varepsilon}, \tilde{r}, \tilde{\varphi}, \tilde{v})$ in $E_{3}$ and for any $\delta_{0}>0$, there exists a subset $\tilde{G}$ of $W$ which is constructed as follows: There exists a $K$-quadrilateral $G$ in $U_{\epsilon_{3}}(\tilde{x}) \cap U_{\epsilon_{2}}(\tilde{x})$ with $\tilde{x} \equiv(\tilde{c}, \tilde{r}, \tilde{\varphi})=\Pi(w)$ such that $T_{*}^{-m_{0}}$ and $T_{*}^{m_{0}}$ are continuous on $G, T_{*}^{-m_{0}} G$ and $T_{*}^{m_{0}} G$ are $K$-quadrilaterals, $\gamma_{a}(G)$ (resp. $\left.\gamma_{b}(G)\right)$ is a segment of the fibre $\gamma^{(c)}(\tilde{x})$ (resp. $\gamma^{(e)}(\tilde{x})$ ), and

$$
\theta\left(\gamma_{a}(G)\right)=\theta\left(\gamma_{b}(G)\right)<\min \left\{\delta_{0}, \delta_{1}, \delta_{2}, \delta_{3}\right\} /\left(1+c_{2}\right) .
$$

Put $\tilde{\gamma}_{a} \equiv \bar{\gamma}^{(+)}(w) \cap \Pi^{-1}(G)$. For $\bar{w}$ in $W$, put

$$
D(\bar{w}) \equiv\left\{\begin{array}{cl} 
& 1-\delta \leq \frac{\varphi-\bar{\varphi}}{r-\bar{r}} \frac{1}{\chi^{(c)}(x)} \leq 1+\delta \\
& f^{(+)}(\tilde{x})-\delta \leq \frac{v-\bar{v}}{\varphi-\bar{\varphi}} \leq f^{(+)}(\tilde{x})+\delta
\end{array}\right\}
$$

with $\bar{w}=(\tilde{c}, \bar{r}, \bar{\varphi}, \bar{v})$. Define $\tilde{G}$ by

$$
\tilde{G}=\bigcup_{-a \leq s \leq a} S_{s}^{f} \bigcup_{\bar{w} \in \tilde{q}_{b}} D(\bar{w}) \cap \Pi^{-1}(G),
$$

with $a=\theta\left(\gamma_{a}(G)\right)$. As stated in $\S 3$, there exist subsets $G^{(c, \delta)}$ and $G^{(e, \tilde{o})}$ of $G$ which satisfy (C-1), (C-2) and (C-3). Put

$$
\tilde{G}^{(c, \delta)} \equiv\left\{w \in \Pi^{-1}\left(G^{(c, \delta)}\right) \cap \tilde{G} ; \tilde{\gamma}^{(+)}(w) \cap \bigcup_{|s| \leq a} S_{s}^{f} \tilde{\gamma}_{b} \neq \phi\right\}
$$

Then one can see the inequality

$$
\mu\left(\tilde{G}^{(e, \delta)} \cap \Pi^{-1}\left(G^{(e, \delta)}\right)\right) \geq(1+2 \delta)^{-4}(1-2 \delta) \mu(\tilde{G}),
$$

since $1-\delta \leq \chi^{(c)}(x) / \chi^{(c)}(\tilde{x}) \leq 1+\delta$ for $x$ in $G$. By the covering theorem of Vitali, there exists a finite family $\mathscr{G}$ of $\tilde{G}$ 's which satisfies

$$
\begin{aligned}
& \mu\left(E_{1} \cap E_{2} \cap E_{3} \cap E_{4} \cap \tilde{G}\right) \geq(1-\delta) \mu(\tilde{G}), \\
& \Pi^{-1}\left(E_{5}\right) \supset \tilde{G}, \\
& \mu\left(E_{1} \cap E_{2} \cap E_{3} \cap E_{4} \cap \Pi^{-1}\left(E_{5}\right)-\bigcup_{\tilde{G} \in \mathcal{g}} \tilde{G}\right)<\delta, \\
& \tilde{G} \cap \tilde{G}^{\prime}=\phi \quad \text { if } \tilde{G} \neq \tilde{G}^{\prime} .
\end{aligned}
$$

Put

$$
E(\tilde{G}) \equiv\left\{w \in \tilde{G}^{(c, \delta)} ; \tilde{\gamma}^{(+)}(w) \cap E_{4} \cap \tilde{G} \neq \phi\right\} .
$$

Then $B \cap E(\tilde{G})$ is $\left.\tilde{\zeta}^{(+)}\right|_{\tilde{G}}$-measurable for every element $B$ of $\bigvee_{k=N^{\prime}}^{N^{\prime \prime}} S_{-k t}^{f} \alpha$ with $N^{\prime \prime}>N^{\prime} \geq 0$. By Lemma 3.1, the estimate 


$$
\begin{aligned}
\mu(B & \left.\cap E(\tilde{G}) \cap \Pi^{-1}\left(G^{(e, \delta)}\right)\right) /(1+\delta) \\
& \leq \int_{\tau a(G) \cap G(e, \delta)} d \rho(\gamma) \int_{\tilde{v}-a-\delta}^{\tilde{v}+a+\delta} d t \int_{B \cap E(\tilde{G}) \cap S_{-}^{\prime} t r b(G)}-\mu_{0} \cos \varphi d \sigma(\varphi) \\
& \leq \mu\left(B \cap E(\tilde{G}) \cap \Pi^{-1}\left(G^{(e, \delta)}\right)\right) /(1-\delta)
\end{aligned}
$$

is obtained. Moreover, for $y$ in $\gamma_{a}(G) \cap G^{(e, \hat{b})}$

$$
1-\delta<\frac{\int_{\tilde{v}-a-2 \delta}^{\tilde{v}+a+2 \delta} d t \int_{B \cap E(\tilde{\theta}) \cap S_{-t}^{f} t^{(e)}(y)} \cos \varphi d \varphi}{\int_{\tilde{v}-a-\delta}^{\tilde{v}+a+\delta} d t \int_{B \cap E(\tilde{Q}) \cap S S_{-t \gamma^{(e)}(y)}} \cos \varphi d \varphi}<1+\delta
$$

is obtained. Therefore, there exists a $\left((1+\delta)^{3}-1\right)$-measure preserving mapping $\phi$ from $\bigcup_{\tilde{\epsilon} \in \mathcal{g}}\left(E(\tilde{G}) \cap \Pi^{-1}\left(G^{(e, \delta)}\right)\right) \cap B$ to $\bigcup_{\tilde{a} \in \boldsymbol{g}}\left(E(\tilde{G}) \cap \Pi^{-1}\left(G^{(e, \delta)}\right)\right)$ such that $\phi$ maps $E(\tilde{G}) \cap \Pi^{-1}\left(\gamma^{(e)}(x)\right) \cap B$ to $\dot{E}(\tilde{G}) \cap \Pi^{-1}\left(\gamma^{(e)}(x)\right)$ for $x$ in $\gamma_{a}(G) \cap G^{(e, \delta)}$. Let $\ell_{i}(w)$ be the name function of $S_{i t}^{f} \alpha$. For $z$ in $E_{1} \cap E_{2}$ $\cap E_{3} \cap E(\tilde{G}) \cap B$

$$
\ell_{i}(z)=\ell_{i}(\phi z) \quad \text { for } 1 \leq i \leq N_{1}-1
$$

and for $n \geq N_{1}$

$$
\frac{1}{n} \sum_{1}^{n} e\left(\ell_{i}(z)-\ell_{i}(\phi z)\right) \leq 2 \delta
$$

hold, since

$$
\begin{aligned}
& \tilde{d}\left(S_{-k t}^{f} z ; \partial \alpha\right)>\delta_{2} \quad \text { for } 1 \leq k \leq N_{1}-1, \\
& \frac{1}{n} \sum_{k=0}^{n-1} I_{E_{1}^{c}}\left(S_{-k t}^{f} z\right) \leq 2 \delta, \\
& \text { (distance of } \left.S_{-k t}^{f} z \text { and } S_{-k t}^{f} \phi z\right) \leq \min \left(\delta_{1}, \delta_{2}, \delta_{3}\right)
\end{aligned}
$$

for $k \geq 0$. On the other hand, there exist an $N_{2}$ and a set $E_{6}$ such that $\mu\left(E_{6}\right)>1-\delta$ and for all $N^{\prime}, N^{\prime \prime} \geq N_{2}$ and all $B$ in $\bigvee_{k=N^{\prime}}^{N^{\prime \prime}} S_{-k t}^{f} \alpha, B \subset E_{6}$,

$$
\left|\mu\left(E_{1} \cap E_{2} \cap E_{3} \cap E(\tilde{G})\right)-\mu\left(E_{1} \cap E_{2} \cap E_{3} \cap E(\tilde{G}) \mid B\right)\right| \leq \delta \mu(\tilde{G})
$$

holds, since $S_{t}^{f}$ is a $K$-system. Hence

$$
\begin{aligned}
& \mu\left(\bigcup_{\tilde{G \in \boldsymbol{g}}}\left(E_{1} \cap E_{2} \cap E_{3} \cap E(\tilde{G}) \cap B\right)\right) \\
& \quad \geq \sum_{\dot{\sigma} \in \boldsymbol{g}} \mu\left(E_{1} \cap E_{2} \cap E_{3} \cap E_{4} \cap \tilde{G}\right) \mu(B)-\delta \mu(B) \\
& \quad \geq(1-\delta) \mu\left(\bigcup_{\tilde{G} \in \boldsymbol{g}} \tilde{G}\right) \mu(B)-\delta \mu(B)
\end{aligned}
$$




$$
\begin{aligned}
& \geq(1-\delta)\left(\mu\left(E_{1} \cap E_{2} \cap E_{3} \cap E_{4} \cap \Pi^{-1}\left(E_{5}\right)\right)-\delta\right) \mu(B)-\delta \mu(B) \\
& \geq[(1-\delta)(1-6 \delta)-\delta] \mu(B) .
\end{aligned}
$$

Therefore by Lemma 2.4, the partition $\alpha$ is very weakly Bernoullian.

Since there exists an increasing sequence of finite partitions $\left\{\alpha_{n}\right\}$ such that $\log \tilde{d}\left(w ; \partial \alpha_{n}\right)$ is integrable and $\alpha_{n}$ increases to $\epsilon$ as $n \rightarrow \infty, S_{t}^{f}$ is a Bernoulli shift for fixed $t \neq 0$.

Q.E.D.

Corollary 6.1. A Sinai billiard system is a Bernoulli flow.

Corollary 6.2. If $\left\{S_{t}^{f}\right\}$ has no point spectrum, then $\left\{S_{t}^{f}\right\}$ is a Bernoulli flow.

Theorem 6. The flow $\left\{S_{t}\right\}$ given in $\S 3$ is a Bernoulli flow, if the assumptions $(\mathrm{H}-1) \sim(\mathrm{H}-3)$ are fulfilled and if $\left\{S_{t}\right\}$ has no point spectrum.

\section{Appendix}

The properties of the partitions $\alpha^{(c)}$ and $\alpha^{(e)}$ have been shown in [5]. Now some of them will be stated. Under the suitable numbering the followings are true, here denote as $a(j)=O\left(j^{b}\right)$ if

$$
0<\lim _{j \rightarrow \infty}|a(j)| j^{-b} \leq \varlimsup_{j \rightarrow \infty}|a(j)| j^{-b}<\infty:
$$

(i) $T_{*} X_{j}^{(e)}=X_{j}^{(c)}$,

(ii) $\tau(x)=O(j) \quad$ for $x \in X_{j}^{(e)}$ (resp. $X_{j}^{(c)}$ ).

Further the following figure is also true.

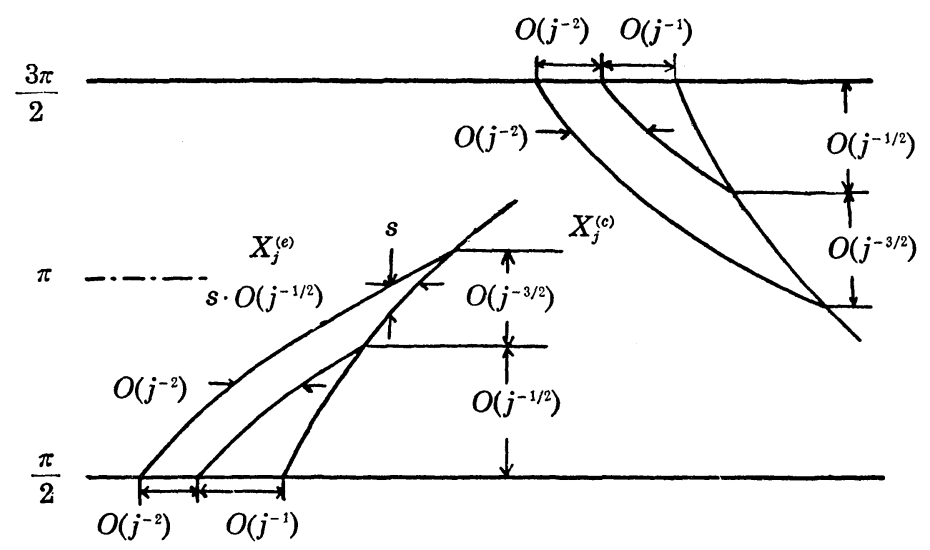

Fig. 2

These show that $\log d\left(x ; \partial \alpha^{(e)}\right)$ is integrable. The condition ( $\left.\mathrm{f}-1\right)$ and (i) imply that the distance between $w=(x, v)$ in $\Pi^{-1}\left(X_{j}^{(e)}\right)$ and the 
boundary $W^{*}$ is greater than $O\left(j^{-1 / 2}\right) d\left(x ; \partial \alpha^{(e)}\right)^{1 / 2}(f(x)-v)$ if $f(x)-v \leq$ $O\left(j^{1 / 2}\right) d\left(x ; \partial \alpha^{(e)}\right)^{1 / 2}$. Moreover,

$$
\sup _{x, y \in X_{j}^{(e)}}(f(x)-f(y)) \leq O(j)
$$

is shown. Hence one can easily obtain that $\log \tilde{d}(w ; \partial \beta)$ is integrable.

\section{REFERENCES}

[1] L. M. Abramov, On the entropy of a flow, Dokl. Akad. Nauk SSSR 128 (1959), 873-876.=Amer. Math. Soc. Transl. (2) 49 (1966), 167-170.

[2] N. A. Friedman and D. S. Ornstein, On isomorphism of weak Bernoulli transformations, Advances in Math. 5 (1970), 365-394.

[ 3 ] G. Gallavotti and D. S. Ornstein, Billiards and Bernoulli schemes, Commun. math. Phys. 38 (1974), 83-101.

[4] Sh. Ito, H. Murata and H. Totoki, Remarks on the isomorphism theorems for weak Bernoulli transformations in general case, Publ. RIMS, Kyoto Univ. 7 (1972), $541-580$.

[5] I. Kubo, Perturbed billiard systems I. The ergodicity of the motion of a particle in a compound central field, Nagoya Math. J. 61 (1976), 1-57.

[6] - The ergodicity of the motion of a particle in a potential field, International symposium on mathematical problems in theoretical physics, Jan. 1975 Kyoto Japan, 141-148.

[ 7 ] D. S. Ornstein, Two Bernoulli shifts with infinite entropy are isomorphic, Advances in Math. 5 (1970), 339-348.

[8] - Imbedding Bernoulli shifts in flows, Lecture Notes in Math. 160 [L. Sucheston (editor): Contribution to ergodic theory and probability] Springer-Verlag (1970), 178-218.

[ 9 ] D. S. Ornstein and B. Weiss, Geodesic flows are Bernoullian, Israel J. Math. 14 (1973), 184-198.

[10] V. A. Rohlin and Ya G. Sinai, Construction and properties of invariant measurable partitions, Dokl. Akad. Nauk SSSR 141 (1961), 1038-1041.=Soviet Math. Dokl. 2 (1961), 1611-1614.

[11] Ya. G. Sinai, On the foundations of the ergodic hypothesis for a dynamical system of statistical mechanics, Dokl. Akad. Nauk SSSR 153 (1963), 1261-1264.=Soviet Math. Dokl. 4 (1963), 1818-1822.

[12] - Dynamical systems with elastic reflections, Uspehi Math. Nauk 25 (1970), 141-192.=Russian Math. Surveys 25 (1970), 137-189.

Nagoya University

Hiroshima University 